\title{
Particle motion between parallel walls: Hydrodynamics and simulation
}

\author{
James W. Swan and John F. Brady \\ Division of Chemistry and Chemical Engineering, California Institute of Technology, \\ Pasadena, California 91125, USA
}

(Received 10 February 2010; accepted 3 August 2010; published online 11 October 2010)

\begin{abstract}
The low-Reynolds-number motion of a single spherical particle between parallel walls is determined from the exact reflection of the velocity field generated by multipoles of the force density on the particle's surface. A grand mobility tensor is constructed and couples these force multipoles to moments of the velocity field in the fluid surrounding the particle. Every element of the grand mobility tensor is a finite, ordered sum of inverse powers of the distance between the walls. These new expressions are used in a set of Stokesian dynamics simulations to calculate the translational and rotational velocities of a particle settling between parallel walls and the Brownian drift force on a particle diffusing between the walls. The Einstein correction to the Newtonian viscosity of a dilute suspension that accounts for the change in stress distribution due to the presence of the channel walls is determined. It is proposed how the method and results can be extended to computations involving many particles and periodic simulations of suspensions in confined geometries. (C) 2010 American Institute of Physics. [doi:10.1063/1.3487748]
\end{abstract}

\section{INTRODUCTION}

The no-slip condition on surfaces constraining a fluid dramatically affects the dynamics of embedded particles, especially at low Reynolds number. Even in the simplest geometries, it is quite difficult to calculate the influence of the boundaries on particle motion. These calculations are important, however, to the understanding of the rheology of microstructured fluids, ${ }^{1}$ the development of microfluidic devices, ${ }^{2}$ and the design and implementation of micro- and nanoscale experiments $^{3}$ among other applications. Study of the complex hydrodynamics resulting from satisfying boundary conditions on both a particle and the boundary surfaces is nearly a century old, but the efficient calculation of these remains a challenge. Building on techniques we developed recently to study the motion of many particles near a single plane wall boundary, ${ }^{4}$ we proceed to calculate the grand mobility tensor associated with a single particle between a set of parallel walls. While other researchers have calculated similar quantities, the results presented here are packaged in a particularly transparent form that identifies the distance between the walls as the key length scale in the problem. We are able to write the contributions to the grand mobility tensor as a finite and ordered sum over inverse powers of the channel width. These expressions are especially useful in computation because they are easily tabulated.

The convergence of colloid science and microfluidics makes the motivation for these calculations apparent. Recent research has been concerned with particle motion in narrow channels via pressure driven flow, electrophoresis, electroosmotic flow, and Brownian motion. The additional resistance generated by channel walls plays a role in regulating the kinetics of colloidal scale-assembly processes ${ }^{5}$ and also affects the dynamics and efficacy of electrophoretic separations. ${ }^{6}$ Additionally, various biological assays work precisely because of the shape of the microfluidic devices in which they are conducted. ${ }^{7}$ Be it Brownian motors, ${ }^{8}$ the deterministic transport of colloids through a varying potential landscape ${ }^{9}$ or the sorting of biological macromolecules in optical lattices, ${ }^{10}$ the fluctuations on which these processes rely are firmly tied to the geometric constraints of the microchannel in which they take place. Invariably in these studies, a statistical description of a microfluidic process that depends on the intimate hydrodynamic details of the particle motion is generated. The present results make the hydrodynamics governing particle motion in a channel easily accessible and may allow researchers to predict readily the outcome of a lab-on-a-chip experiment.

Some time ago, Faxén ${ }^{11}$ approached the problem of particle motion between parallel walls by noting the similarities between Laplace's equation and the Stokes equations for fluid flow at low Reynolds number, which for a fluid of viscosity $\eta$ with velocity field $\mathbf{v}$ and pressure $p$ are

$$
\begin{aligned}
& \nabla^{2} \mathbf{v}=\frac{1}{\eta} \nabla p, \\
& \nabla \cdot \mathbf{v}=0 .
\end{aligned}
$$

Expressing the fundamental solution to Laplace's equation in three dimensions $(1 / r)$ as an integral, he wrote down the general solution to the Stokes equations between a set of parallel walls in integral form. Here, we produce an equivalent result using the direct process of transforming the Stokes equations from real space to Fourier space; after all, Faxén's procedure yields a general solution to the Stokes equations in Fourier space. Inverting this solution is rather difficult for all but a few specific geometric configurations that Faxén, to his credit, was able to interrogate. The details of this calculation are available in the text by Happel and Brenner. ${ }^{12}$ Before solving the parallel wall problem, Faxén computed the resistance to the motion of a spherical particle in the half-space 
above a single plane wall. In order to forego the difficulties associated with satisfying the boundary conditions on the two walls lining a channel, Oseen ${ }^{13}$ suggested that a linear superposition of the resistance due to each plane wall separately would make for a suitable estimate. This approach has seen some empirical success, but the approximation made can be quite substantial. It poses a severe computational challenge as it fails to yield a positive-definite grand mobility tensor-something that is essential to the physics of Stokes flow and critical for the computation of hydrodynamic interactions among many particles.

Blake $^{14}$ brought an electrostatic perspective and solved the problem of a point force in the half-space above a single plane wall in Stokes flow. He introduced the notion of an image flow below the plane wall that satisfies the Stokes equations and cancels the flow due to the point force exactly where the plane wall bounds the half-space. The proper manipulation of this expression has proven quite useful recently in the simulation of many particles near a plane wall boundary. ${ }^{4}$ Following on Blake's approach, Liron and Mochon ${ }^{15}$ found that an infinite but convergent series of hydrodynamic images was necessary to satisfy the boundary conditions on two plane walls with a point force between them. Summation of this series is possible but ineffective for rapid simulation of particle dynamics. However, this result helps us understand why inversion of Faxén's Fourier space results into an analytical, real space result like Blake's proves so difficult: since the Stokes equations are unique, the result must be the same as Liron and Mochon's infinite sums, which themselves are quite complicated.

There have been a number of attempts to simulate suspensions of spherical particles between parallel walls. Durlofsky and Brady ${ }^{16}$ discretized the force density on the walls and used this to calculate the effects of the walls on a finite set of particles embedded in the fluid. One approach used spherical particles fixed in space as a model of channel walls in plane Couette flow. ${ }^{17,18}$ Both of these methods model the channel walls as plane surfaces which are, for lack of better terms, "leaky" and "slippery." There has been much success ${ }^{19-21}$ using Fourier transform techniques in addition to an eigenfunction expansion of the solution to Stokes flow in the parallel wall geometry to calculate the image flows due to two walls. While this result must be the same as Liron and Mochon's, it satisfies the boundary conditions on the walls at each level of the eigenfunction expansion, which is a clear advantage. Their expansion, however, does not make a direct physical connection to the moments of the force density on the particle's surface, and also has an implicit dependence of the solution on the channel width. Other approaches to the computation of the channel mobility have similar features (boundary collocation method, ${ }^{22}$ boundary integral method $^{23}$ ). Our approach utilizes a physical connection to the force moments of a particle between parallel walls, which may afford novel, more intuitive, and applicable results.

A number of researchers have made experimental measurements of the in-plane diffusivity of a particle between parallel walls. Dufresne et al. ${ }^{24}$ did a thorough job of comparing many of the analytical approaches to the diffusivity of a single particle in a channel measured via optical tweezer microscopy. They find that all of the approaches, while giving different results, fall equally near the experimental data and well within the margins of error. Unfortunately like previous studies, these disappoint when it comes to decoupling the three separate length scales in the problem: the characteristic size of the particle, $a$; the separation between the channel walls, $H$; and the height of the particle above one of the channel walls, $h$. Distinguishing clearly among these is essential to understanding how many particles behave in a bounded geometry. As Stokes flows tend to decay slowly, the effects of interactions with the walls are often just as important as those between particles themselves. We attempt to remedy this shortcoming here.

The paper is organized as follows. In Sec. II we detail the development of the grand mobility tensor from Faxén formulas and multipole expansions and briefly discuss the relevant velocity fields for flow between parallel walls. We introduce the Fourier transform solution to the Stokes equations between parallel walls and demonstrate that this can be used to develop an integral expression for components of the grand mobility tensor for a single particle in a channel. In particular, we show that each element of the grand mobility tensor can be written in terms of inverse powers of the channel width. In Sec. III we plot the elements of the grand mobility tensor for coupling between translation, rotation, and rate of strain with force, torque, and stresslet. We also show how these collapse down to the single wall results in the limit that the channel is infinitely wide. We use a Stokesian dynamics simulation to calculate the fall speed and rate of rotation of a particle as it sediments down a channel. We use this same simulation to calculate the Brownian drift of a particle between parallel walls. Finally, we calculate an extension of the Einstein correction for the shear viscosity of a dilute colloidal suspension which accounts for the effects of the channel walls on the distribution of stresses in the channel. In Sec. IV, we discuss the extension of these results to the study of many particles between parallel walls. In particular, we discuss how a similar approach may be fruitful in constructing Stokesian dynamics and accelerated Stokesian dynamics simulations of infinite suspensions bound between parallel walls.

\section{THEORY AND METHODS}

\section{A. The grand mobility tensor}

For Stokes flow surrounding rigid particles and contained by rigid boundaries, the governing equations and boundary conditions are linear in the velocity field and boundary data. Taking a higher level perspective on the problem than the detailed knowledge of the velocity field, we recognize that the translational velocities of the particles between the walls must be coupled linearly to forces on the particles. If we generalize a bit more, then we can allow that the translational $\left(\mathbf{U}-\mathbf{U}^{\infty}\right)$ and rotational $\left(\boldsymbol{\Omega}-\boldsymbol{\Omega}^{\infty}\right)$ velocities of the particles relative to the bulk fluid velocity as well as the rate of strain of the fluid $\left(\mathbf{E}^{\infty}\right)$ at the particles' center and higher order moments of the velocity distribution in the fluid couple linearly to all the moments of force density on the particles' surface. These include, but are hardly limited to, 
the hydrodynamic force $\mathbf{F}^{H}$, the hydrodynamic torque $\mathbf{L}^{H}$, and the hydrodynamic stresslet $\mathbf{S}^{H}$. The latter two represent the antisymmetric and symmetric parts of the first moment of the force density. This linearity may be expressed in a very simple and compact form,

$$
\left(\begin{array}{c}
\mathbf{U}-\mathbf{U}^{\infty} \\
\mathbf{\Omega}-\mathbf{\Omega}^{\infty} \\
-\mathbf{E}^{\infty} \\
\vdots
\end{array}\right)=-\left(\begin{array}{cccc}
\mathbf{M}_{U F} & \mathbf{M}_{U L} & \mathbf{M}_{U S} & \cdots \\
\mathbf{M}_{\Omega F} & \mathbf{M}_{\Omega L} & \mathbf{M}_{\Omega S} & \cdots \\
\mathbf{M}_{E F} & \mathbf{M}_{E L} & \mathbf{M}_{E S} & \cdots \\
\vdots & \vdots & \vdots & \ddots
\end{array}\right) \cdot\left(\begin{array}{c}
\mathbf{F}^{H} \\
\mathbf{L}^{H} \\
\mathbf{S}^{H} \\
\vdots
\end{array}\right)
$$

where M's are the mobility tensors. These depend only on the geometry defined by the positions of all the particles relative to the boundaries and are independent of the velocity and force moments. When the mobility tensors are assembled in this way, the construction is termed the grand mobility tensor and is sometimes given the designation $\mathcal{M}$. By using the Lorentz reciprocal theorem and dissipation arguments, it is easy to show that, regardless of the boundary geometries, the grand mobility tensor is symmetric and positive-definite. $^{25}$ These properties are fundamental to Stokes flow and are often quite useful when simulating the motion of particles at low Reynolds number. We will restrict the discussion for the remainder of the paper to spherical particles as these are easiest to deal with mathematically. Though, similar expressions exist for any particle or boundary geometry.

Faxén formulas are often used to assemble the mobility tensors. These relate, for instance, the relative velocity of a spherical particle with center at $\mathbf{x}_{0}$ in an unbounded fluid to the force on that particle and the effects of a disturbance flow denoted $\mathbf{v}^{\prime}(\mathbf{x})$, viz.,

$$
\mathbf{U}-\mathbf{U}^{\infty}=-\frac{\mathbf{F}^{H}}{6 \pi \eta a}+\left.\left(1+\frac{a^{2}}{6} \nabla_{x}^{2}\right) \mathbf{v}^{\prime}(\mathbf{x})\right|_{\mathbf{x}=\mathbf{x}_{0}} .
$$

If we knew the exact Green's function for a particle between two walls, then we could derive an equivalent Faxén formula for a particle in a channel. All that would change in the above formula would be the first term on the right hand side. As we shall see, however, this is unnecessary since the velocity disturbance caused by a single particle in a channel can be divided into two pieces: the flow due to the particle as though in an unbounded fluid, and the correction to that flow, which cancels on the boundaries and satisfies the relevant boundary conditions. This second velocity field, termed the reflection, is nothing more than a disturbance flow which contributes to $\mathbf{v}^{\prime}(\mathbf{x})$. Equivalent formulas exist that couple the relative rotation and rate of strain to the torque and stresslet, respectively, as well as to a disturbance flow, viz.,

$$
\boldsymbol{\Omega}-\boldsymbol{\Omega}^{\infty}=-\frac{\mathbf{L}^{H}}{8 \pi \eta a^{3}}+\frac{1}{2} \nabla_{x} \times\left.\mathbf{v}^{\prime}(\mathbf{x})\right|_{\mathbf{x}=\mathbf{x}_{0}},
$$

$$
\begin{aligned}
-\mathbf{E}^{\infty}= & -\frac{\mathbf{S}^{H}}{\frac{20}{3} \pi \eta a^{3}}+\frac{1}{2}\left(1+\frac{a^{2}}{10} \nabla_{x}^{2}\right) \\
& \times\left.\left[\nabla_{x} \mathbf{v}^{\prime}(\mathbf{x})+{ }^{T} \nabla_{x} \mathbf{v}^{\prime}(\mathbf{x})\right]\right|_{\mathbf{x}=\mathbf{x}_{0}},
\end{aligned}
$$

where ${ }^{T} \nabla_{x}$ is the front-gradient-transpose operator that takes the dyadic gradient and transposes it with the first index of the operand. Higher order Faxén formulas can be derived using procedures like those described in the classic texts on Stokes flow. ${ }^{12,25}$ The three we have presented here are adequate for illustrating our methods and results.

Since we are seeking to build the mobility tensors for a particle in a channel, we need to determine the reflected flow mentioned above. To do this, we first establish the velocity field generated by a spherical particle in an unbounded flow with one of the given force multipoles on its surface. This is most easily done with a multipole expansion. Given the fundamental point force solution to Stokes flow in an unbounded domain, called the Stokeslet,

$$
\mathbf{J}(\mathbf{r})=\frac{1}{8 \pi \eta}\left(\frac{\mathbf{I}}{r}+\frac{\mathbf{r r}}{r^{3}}\right),
$$

the velocity field surrounding a rigid, no-slip particle is written as

$$
\mathbf{v}(\mathbf{x})=\int_{S} \mathbf{J}(\mathbf{x}-\mathbf{y}) \cdot \mathbf{f}(\mathbf{y}) d S_{y},
$$

where $S$ designates the surface of the particle and $\mathbf{f}(\mathbf{y})$ is the force density on the particle's surface. If we perform a Taylor expansion of the Stokeslet about the particle's center, we can write the velocity field generated by the particle as

$$
\begin{aligned}
\mathbf{v}(\mathbf{x})= & \left.\left(1+\frac{a^{2}}{6} \nabla_{y}^{2}\right) \mathbf{J}(\mathbf{x}-\mathbf{y})\right|_{\mathbf{y}=\mathbf{x}_{0}} \cdot \mathbf{F}+\frac{1}{2} \nabla_{y} \\
& \times\left.\mathbf{J}(\mathbf{x}-\mathbf{y})\right|_{\mathbf{y}=\mathbf{x}_{0}} \cdot \mathbf{L}+\frac{1}{2}\left(1+\frac{a^{2}}{10} \nabla_{y}^{2}\right) \\
& \times\left.\left(\nabla_{y}+\nabla_{y}^{T}\right) \mathbf{J}(\mathbf{x}-\mathbf{y})\right|_{\mathbf{y}=\mathbf{x}_{0}}: \mathbf{S}+\cdots,
\end{aligned}
$$

where $\nabla_{x}^{T}$ is the back-gradient transpose operator which takes the dyadic gradient and transposes it with the last index of the operand. Here, we have switched from referring to the force, torque, and stresslet on the fluid to the force moments on the particle which may be represented as $\mathbf{F}=-\mathbf{F}^{H}$, $\mathbf{L}=-\mathbf{L}^{H}$, and $\mathbf{S}=-\mathbf{S}^{H}$. This series continues with terms that are represented as higher order derivatives of the Stokeslet and higher order force moments. These higher order terms all decay faster than the ones retained since the Stokeslet itself decays as $1 / r$. This means that for relatively large separations, only a few force multipoles are necessary to accurately represent the flow. We are free to specify any force density on the particle's surface, and therefore the velocity field generated by a spherical particle with a constant force density on its surface in an unbounded fluid is simply 


$$
\mathbf{v}(\mathbf{x})=\left.\left(1+\frac{a^{2}}{6} \nabla_{y}^{2}\right) \mathbf{J}(\mathbf{x}-\mathbf{y})\right|_{\mathbf{y}=\mathbf{x}_{0}} \cdot \mathbf{F} .
$$

Knowing this is crucial to deriving the reflection of the unbounded flow off all channel walls and subsequently applying the appropriate Faxén formula.

It will prove convenient to define the flow due just to a Stokeslet of magnitude $\mathbf{F}$ originating at a point $\mathbf{y}$ in an otherwise unbounded fluid as

$$
\mathbf{v}^{S}(\mathbf{x} ; \mathbf{y})=\mathbf{J}(\mathbf{x}-\mathbf{y}) \cdot \mathbf{F} .
$$

The reflection of this Stokeslet off the channel walls must satisfy the Stokes equations between the walls as well as cancel the Stokeslet flow on the channel walls themselves. We denote the reflection flow as $\mathbf{v}^{S^{\prime}}(\mathbf{x} ; \mathbf{y})$ and write the boundary condition on the channel walls in the most primitive form,

$$
\mathbf{v}^{S^{\prime}}(\mathbf{x} ; \mathbf{y})+\mathbf{v}^{S}(\mathbf{x} ; \mathbf{y})=0, \quad \text { for } \mathbf{x} \in \text { walls. }
$$

We will establish a defined geometry in Sec. II C; however, here we aim to layout the construction of the mobility tensor using reflected flows. Referring back to the flow generated by a particle with a constant force density on its surface [Eq. (9)], we see that the reflected flow, designated $\mathbf{v}^{\prime}(\mathbf{x})$, must satisfy the boundary conditions,

$$
\mathbf{v}^{\prime}(\mathbf{x})+\left.\left(1+\frac{a^{2}}{6} \nabla_{y}^{2}\right) \mathbf{J}(\mathbf{x}-\mathbf{y})\right|_{\mathbf{y}=\mathbf{x}_{0}} \cdot \mathbf{F}=0,
$$

where $\mathbf{x}$ is a point on the walls. We use Eq. (11) and the Laplacian with respect to $\mathbf{y}$ of that same equation to argue that the constant-force-particle reflection flow can be written in terms of the Stokeslet reflection flow,

$$
\mathbf{v}^{\prime}(\mathbf{x})=\left.\left(1+\frac{a^{2}}{6} \nabla_{y}^{2}\right) \mathbf{v}^{S^{\prime}}(\mathbf{x} ; \mathbf{y})\right|_{\mathbf{y}=\mathbf{x}_{0}} .
$$

This satisfies the wall boundary condition in Eq. (12) exactly. Similar expressions hold for other multipole generated velocity fields, and we find fortuitously that we only need to work out the reflection of the Stokeslet off the channel walls to determine all the other flows that the particle might generate. Additionally, we recognize from the boundary condition that the flow $\mathbf{v}^{S^{\prime}}(\mathbf{x} ; \mathbf{y})$ must be linear in the forcing $\mathbf{F}$. Treating $\mathbf{v}^{\prime}(\mathbf{x})$ as a disturbance velocity and referring back to the Faxén formulas, the relative translational velocity of a particle in a channel can be written as

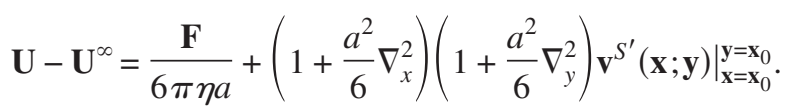

This is the mobility tensor $\mathbf{M}_{U F}$ for a particle in a channel, and similar expressions can be developed for the other pieces of the grand mobility tensor. ${ }^{4}$ In Sec. II C, we complete the development of these tensors by determining a general equation for the Stokes flow in a channel with arbitrary boundary conditions on the walls.

\section{B. General solution to the Stokes equations between parallel walls}

Given the Stokes equations [Eq. (1)] and a pair of boundary conditions on the lower and upper walls of the channel represented as

$$
\begin{aligned}
& \mathbf{v}(\mathbf{x})=\mathbf{v}^{L}(\mathbf{x}), \quad \text { for } \mathbf{x} \in \text { lower wall, } \\
& \mathbf{v}(\mathbf{x})=\mathbf{v}^{U}(\mathbf{x}), \quad \text { for } \mathbf{x} \in \text { upper wall, }
\end{aligned}
$$

we seek a general solution for $\mathbf{v}(\mathbf{x})$. Taking cues from Faxén and Blake, we first find a solution in Fourier space by transforming the coordinates parallel to the wall; henceforth designated $r_{1}$ and $r_{2}$ with unit vectors $\boldsymbol{\delta}_{1}$ and $\boldsymbol{\delta}_{2}$. We use the following Fourier transform and inverse in this process,

$$
\begin{aligned}
& \hat{\zeta}=\mathcal{F}(\zeta)=\iint_{-\infty}^{\infty} e^{i\left(k_{1} r_{1}+k_{2} r_{2}\right)} \zeta d r_{1} d r_{2}, \\
& \zeta=\mathcal{F}^{-1}(\hat{\zeta})=\frac{1}{(2 \pi)^{2}} \iint_{-\infty}^{\infty} e^{-i\left(k_{1} r_{1}+k_{2} r_{2}\right)} \hat{\zeta} d k_{1} d k_{2} .
\end{aligned}
$$

This transform leaves unchanged the coordinate perpendicular to the walls which we designate as $r_{3}$ with unit vector $\boldsymbol{\delta}_{3}$. Therefore, transforming the Stokes equations and noting that in Stokes flow the pressure is also harmonic, the governing equations are reduced to a set of ordinary differential equations which depend only on the reciprocal coordinates $k_{1}$ and $k_{2}$ and the real space coordinate $r_{3}$,

$$
\begin{aligned}
& -k^{2} \hat{p}+\frac{\partial^{2} \hat{p}}{\partial r_{3}^{2}}=0, \\
& -k^{2} \hat{v}_{i}+\frac{\partial^{2} \hat{v}_{i}}{\partial r_{3}^{2}}=\frac{1}{\eta}\left(-i k_{\alpha} \delta_{i \alpha} \hat{p}+\frac{\partial \hat{p}}{\partial r_{3}} \delta_{i 3}\right), \\
& -i k_{\alpha} \hat{v}_{\alpha}+\frac{\partial \hat{v}_{3}}{\partial r_{3}}=0,
\end{aligned}
$$

where $k^{2}=k_{1}^{2}+k_{2}^{2}$. Blake ${ }^{14}$ first derived these expressions in his study of the Stokeslet above a single wall in an otherwise unbounded half-space. Here, we use two different summation notations where Greek indices (e.g., $\alpha, \beta$ ) can assume the values $(1,2)$ while Roman indices (e.g., $i, j)$ can assume the values $(1,2,3)$. Additionally, repeated indices signal the usual summation over all attainable index values. The general solutions for the pressure and the velocity field can be written as

$$
\begin{aligned}
\hat{p}= & A e^{-r_{3} k}+B e^{r_{3} k}, \\
\hat{\mathbf{v}}= & \mathbf{A} e^{-r_{3} k}+\mathbf{B} e^{r_{3} k}+\frac{1}{4 \eta k^{2}}\left[A \mathbf{d}\left(2 r_{3} k+1\right) e^{-r_{3} k}\right. \\
& \left.+B \overline{\mathbf{d}}\left(2 r_{3} k-1\right) e^{r_{3} k}\right],
\end{aligned}
$$




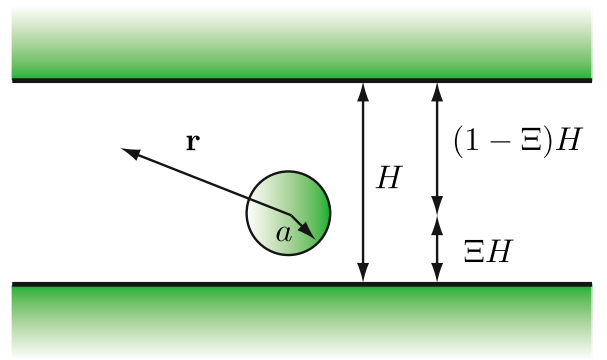

FIG. 1. (Color online) A single spherical particle of radius $a$ in a channel of width $H$. The vector $\mathbf{r}$ is centered on the particle which lies a fractional distance $\Xi$ across the channel.

where $d_{i}=i k_{\alpha} \delta_{i \alpha}+k \delta_{i 3}$ and $\bar{d}_{i}=-i k_{\alpha} \delta_{i \alpha}+k \delta_{i 3}$ are related by conjugation. The unknown coefficients $A, B, \mathbf{A}$, and $\mathbf{B}$ are related through the equation of continuity [Eq. (21)] by

$$
A=2 \eta \mathbf{d} \cdot \mathbf{A},
$$

$$
B=-2 \eta \overline{\mathbf{d}} \cdot \mathbf{B} .
$$

The remaining vectors $\mathbf{A}$ and $\mathbf{B}$ are determined directly from applying the boundary conditions on the lower and upper walls and depend only on the geometry and the reciprocal coordinates. This process is complicated mathematically and involves solving a coupled set of equations for $A$ and $B$, but the result is an equation for the Fourier transform of the Stokes velocity field in a channel given arbitrary boundary conditions on the walls. We work this out explicitly in the Appendix for posterity. Of course, what is really needed for determining the mobility tensors for a spherical particle between a pair of walls is the real space solution to these equations. In Sec. II C, we address this issue.

\section{Single particle mobility in a channel}

Up to this point, we have avoided writing down any specific geometry associated with the system in order to keep the analysis as general as possible. From here on, where the particle is located in the channel as well as the channel width will need to be specified. This will provide a proper origin to the coordinates in Sec. II B. Figure 1 details the geometry of the problem. The spherical particle of radius $a$ lies a distance $\Xi H$ above the lower wall which itself is a distance $H$ away from the upper wall. The coordinates $\left(r_{1}, r_{2}, r_{3}\right)$ now have a natural origin corresponding to the center of the particle. This means that the lower wall corresponds to $r_{3}=-\Xi H$ and the upper wall corresponds to $r_{3}=(1-\Xi) H$.

As a first step, the reflection of the Stokeslet field originating from the center of the particle, $\mathbf{v}^{S^{\prime}}(\mathbf{r})$, is found. In reciprocal space, the boundary conditions on the Stokeslet reflection are given by

$$
\begin{aligned}
& \hat{\mathbf{v}}^{S^{\prime}}\left(k_{1}, k_{2},-\Xi H\right)=-\hat{\mathbf{v}}^{S}\left(k_{1}, k_{2},-\Xi H\right), \\
& \hat{\mathbf{v}}^{S^{\prime}}\left[k_{1}, k_{2},(1-\Xi) H\right]=-\hat{\mathbf{v}}^{S}\left[k_{1}, k_{2},(1-\Xi) H\right],
\end{aligned}
$$

where the Fourier transform of the Stokeslet is

$$
\begin{aligned}
\hat{\mathbf{v}}^{S}\left(k_{1}, k_{2}, r_{3}\right)= & -\frac{1}{4 \eta k^{3}}\left[\mathbf{I}\left(\frac{\partial^{2}}{\partial r_{3}^{2}}-k^{2}\right)+\frac{1}{(2 k)^{2}} \mathbf{d d}\right. \\
& \times\left(2 k \frac{\partial}{\partial r_{3}}-\frac{\partial^{2}}{\partial r_{3}^{2}}-k^{2}\right)+\frac{1}{(2 k)^{2}} \overline{\mathbf{d}} \mathbf{d} \\
& \times\left(-\frac{\partial^{2}}{\partial r_{3}^{2}}+k^{2}\right)+\frac{1}{(2 k)^{2}} \mathbf{d} \overline{\mathbf{d}}\left(-\frac{\partial^{2}}{\partial r_{3}^{2}}+k^{2}\right) \\
& \left.-\frac{1}{(2 k)^{2}} \overline{\mathbf{d}} \overline{\mathbf{d}}\left(2 k \frac{\partial}{\partial r_{3}}+\frac{\partial^{2}}{\partial r_{3}^{2}}+k^{2}\right)\right] \\
& \cdot \mathbf{F}\left(1+k\left|r_{3}\right|\right) e^{-\left|r_{3}\right| k} .
\end{aligned}
$$

We write this in a form suitable for our particular method, but again this result was first realized by Blake ${ }^{14}$ in his previously mentioned study. We proceed by applying the boundary conditions and finding the unknown coefficients $\mathbf{A}$ and $\mathbf{B}$ associated with the Stokes flow reflection problem. These are now complicated functions of the reciprocal coordinates $\left(k_{1}, k_{2}\right)$, the separation between the plates $(H)$, and the fractional distance across the channel $(\Xi)$. They are also linear functions of the force or Stokeslet magnitude $\mathbf{F}$.

Computing the inverse transform of $\hat{\mathbf{v}}^{S^{\prime}}$ is quite difficult. However, to compute the mobility of a particle in the channel, we really only need to find the value of reflected field at the particle's center which may be written as

$$
\mathbf{v}^{S^{\prime}}\left(\mathbf{x}_{0} ; \mathbf{x}_{0}\right)=\frac{1}{(2 \pi)^{2}} \iint_{-\infty}^{\infty} \hat{\mathbf{v}}^{S^{\prime}}\left(k_{1}, k_{2}, 0\right) d k_{1} d k_{2} .
$$

This integral is orders of magnitude easier to compute than the full inverse Fourier transform since the integrand depends only on a few parameters $\left(k_{1}, k_{2}, \Xi, H\right.$, and $\left.\mathbf{F}\right)$. In fact, we also need to know $\nabla_{x}^{2} \mathbf{v}^{S^{\prime}}(\mathbf{x} ; \mathbf{y}), \nabla_{y}^{2} \mathbf{v}^{S^{\prime}}(\mathbf{x} ; \mathbf{y})$, and $\nabla_{x}^{2} \nabla_{y}^{2} \mathbf{v}^{S^{\prime}}(\mathbf{x} ; \mathbf{y})$ with $\mathbf{x}$ and $\mathbf{y}$ at the center of the particle in order to apply the Faxén formula and to compute $\mathbf{M}_{U F}$. Other higher order derivatives are also necessary to compute higher order mobility tensors. Computing these derivatives is quite easy, however. First, we write down the gradients with respect to the coordinates $\mathbf{x}$ and $\mathbf{y}$ in terms of the coordinate system we have established in the channel $(\mathbf{r}=\mathbf{x}-\mathbf{y})$. In this case, $\mathbf{x}$ refers to some point in the fluid while $\mathbf{y}$ refers to the origin of the coordinate system. These gradients can be written as

$$
\begin{aligned}
& \nabla_{x}=\nabla_{r}, \\
& \nabla_{y}=-\nabla_{r}+\frac{1}{H} \frac{\partial}{\partial \Xi} \boldsymbol{\delta}_{3},
\end{aligned}
$$

and higher order derivatives can be computed by successive application of these formulas. The correct transformation of 
the gradients with respect to $\mathbf{y}$ is important because these derivatives reflect the key point: that the force density is distributed over the particle's surface rather than originating at its center. Failing to note this will result in a grand mobility tensor which is an approximation to the correct result, but fails to be symmetric and positive-definite. Now, we can compute, for instance, $\nabla_{x}^{2} \mathbf{v}^{s^{\prime}}(\mathbf{x} ; \mathbf{y})$ with $\mathbf{x}$ and $\mathbf{y}$ pointing at the particle's center as

$$
\begin{aligned}
\left.\nabla_{x}^{2} \mathbf{v}^{S^{\prime}}(\mathbf{x} ; \mathbf{y})\right|_{\substack{\mathbf{x}=\mathbf{x}_{0} \\
\mathbf{y}=\mathbf{x}_{0}}} & \frac{1}{(2 \pi)^{2}} \iint_{-\infty}^{\infty}\left(-k^{2}+\frac{\partial^{2}}{\partial r_{3}^{2}}\right) \hat{\mathbf{v}}^{S^{\prime}} \\
& \times\left.\left(k_{1}, k_{2}, r_{3}\right)\right|_{r_{3}=0} d k_{1} d k_{2},
\end{aligned}
$$

where we have carefully applied the usual Fourier transform identities to move the derivatives inside the integral. The other terms are computed similarly, and although the formulas are tedious, they yield similar results. The total inverse Fourier transform needed to compute $\mathbf{M}_{U F}$ is given by

$$
\begin{aligned}
& \frac{1}{(2 \pi)^{2}} \iint_{-\infty}^{\infty}\left[1+\frac{a^{2}}{6}\left(-k^{2}+\frac{\partial^{2}}{\partial r_{3}^{2}}\right)\right]\left[1+\frac{a^{2}}{6}\left(-k^{2}+\frac{\partial^{2}}{\partial r_{3}^{2}}\right.\right. \\
& \left.\left.-\frac{2}{H} \frac{\partial^{2}}{\partial \Xi \partial r_{3}}+\frac{1}{H^{2}} \frac{\partial^{2}}{\partial \Xi^{2}}\right)\right]\left.\hat{\mathbf{M}}\left(k_{1}, k_{2}, r_{3} ; \Xi, H\right)\right|_{r_{3}=0} d k_{1} d k_{2},
\end{aligned}
$$

where

$$
\hat{\mathbf{v}}^{S^{\prime}}\left(k_{1}, k_{2}, r_{3}\right)=\hat{\mathbf{M}}\left(k_{1}, k_{2}, r_{3} ; \Xi, H\right) \cdot \mathbf{F}
$$

takes advantage of the linear dependence of the velocity on the forcing. As it happens, the reciprocal length scale $k$ and the channel width $H$ always appear together in the formula for $\hat{\mathbf{M}}$. By redefining the variables of integration in Eq. (33) so that $\xi_{1}=k_{1} H, \xi_{2}=k_{2} H$, and $\xi^{2}=(k H)^{2}$, we integrate over $\xi_{1}$ and $\xi_{2}$ in cylindrical polar coordinates; performing the angular integration analytically and the radial integration numerically. This is quite simple as the integrand decays exponentially as $\xi$ gets large. This yields an expression for $\mathbf{M}_{U F}$ which is an ordered sum of inverse powers of the channel width. The mobility tensor can also be separated into two pieces reflecting motions parallel and perpendicular to the walls, viz.,

$$
\begin{aligned}
\mathbf{M}_{U F}= & \frac{1}{6 \pi \eta a}\left\{( \mathbf { I } - \boldsymbol { \delta } _ { 3 } \boldsymbol { \delta } _ { 3 } ) \left[1-f_{1}^{(U F)}(\Xi)\left(\frac{a}{H}\right)+f_{3}^{(U F)}(\Xi)\right.\right. \\
& \left.\times\left(\frac{a}{H}\right)^{3}-f_{5}^{(U F)}(\Xi)\left(\frac{a}{H}\right)^{5}\right]+\boldsymbol{\delta}_{3} \boldsymbol{\delta}_{3}\left[1-g_{1}^{(U F)}(\Xi)\right. \\
& \left.\left.\times\left(\frac{a}{H}\right)+g_{3}^{(U F)}(\Xi)\left(\frac{a}{H}\right)^{3}-g_{5}^{(U F)}(\Xi)\left(\frac{a}{H}\right)^{5}\right]\right\},(35)
\end{aligned}
$$

where $\mathbf{I}$ is the idem tensor and $f_{i}^{(U F)}(\Xi)$ and $g_{i}^{(U F)}(\Xi)$ are functions of the fractional distance across the channel only. From a far-field perspective, the channel width can be decoupled from the fractional distance across the channel. This means that the functions $f_{i}^{(U F)}(\Xi)$ and $g_{i}^{(U F)}(\Xi)$ need only be computed once for all $\Xi \in(0,1)$ and the hydrodynamic interactions between the particle and the wall are determined completely for all channel widths. This is especially useful in simulations because these functions can be tabulated and referenced quickly.

Similar expressions exist for the other mobility tensors. The Faxén formulas and multipolar flows needed to compute these terms are given explicitly in the paper by Swan and Brady. ${ }^{4}$ We show the result of taking these derivatives and then integrating the result here. Since the grand mobility tensor is symmetric, we only provide six of the nine tensors in Eq. (2). The other three can be computed directly by accounting for this symmetry. For the translation-torque and translation-stresslet couplings, we find the following expressions:

$$
\begin{aligned}
\mathbf{M}_{U L}= & \frac{1}{6 \pi \eta a^{2}} \boldsymbol{\delta}_{3} \cdot \boldsymbol{\epsilon}\left[f_{2}^{(U L)}(\Xi)\left(\frac{a}{H}\right)^{2}+f_{4}^{(U L)}(\Xi)\left(\frac{a}{H}\right)^{4}\right], \\
\mathbf{M}_{U S}= & \frac{1}{6 \pi \eta a^{2}}\left\{[ ( \mathbf { I } - \boldsymbol { \delta } _ { 3 } \boldsymbol { \delta } _ { 3 } ) \boldsymbol { \delta } _ { 3 } + ( \mathbf { I } - \boldsymbol { \delta } _ { 3 } \boldsymbol { \delta } _ { 3 } ) \boldsymbol { \delta } _ { 3 } ^ { T } ] \left[-f_{2}^{(U S)}(\Xi)\right.\right. \\
& \left.\times\left(\frac{a}{H}\right)^{2}+f_{4}^{(U S)}(\Xi)\left(\frac{a}{H}\right)^{4}-f_{6}^{(U S)}(\Xi)\left(\frac{a}{H}\right)^{6}\right] \\
& +\left[\boldsymbol{\delta}_{3}\left(\mathbf{I}-\boldsymbol{\delta}_{3} \boldsymbol{\delta}_{3}\right)+2 \boldsymbol{\delta}_{3} \boldsymbol{\delta}_{3} \boldsymbol{\delta}_{3}\right]\left[g_{2}^{(U S)}(\Xi)\left(\frac{a}{H}\right)^{2}-g_{4}^{(U S)}\right. \\
& \left.\left.\times(\Xi)\left(\frac{a}{H}\right)^{4}+g_{6}^{(U S)}(\Xi)\left(\frac{a}{H}\right)^{6}\right]\right\},
\end{aligned}
$$

where $\boldsymbol{\epsilon}$ is the Levi-Civita tensor. Similarly, for the rotationtorque and rotation-stresslet couplings, the elements of the grand mobility tensor are

$$
\begin{aligned}
\mathbf{M}_{\Omega L}= & \frac{1}{6 \pi \eta a^{3}}\left\{\left(\mathbf{I}-\boldsymbol{\delta}_{3} \boldsymbol{\delta}_{3}\right)\left[\frac{3}{4}-f_{3}^{(\Omega L)}(\Xi)\left(\frac{a}{H}\right)^{3}\right]\right. \\
& \left.+\boldsymbol{\delta}_{3} \boldsymbol{\delta}_{3}\left[\frac{3}{4}-g_{3}^{(\Omega L)}(\Xi)\left(\frac{a}{H}\right)^{3}\right]\right\}, \\
\mathbf{M}_{\Omega S}= & \frac{1}{6 \pi \eta a^{3}}\left(\boldsymbol{\delta}_{3} \cdot \boldsymbol{\epsilon} \boldsymbol{\delta}_{3}+\boldsymbol{\delta}_{3} \cdot \boldsymbol{\epsilon} \boldsymbol{\delta}_{3}^{T}\right) \\
& \times\left[f_{3}^{(\Omega S)}\left(\frac{a}{H}\right)^{3}+f_{5}^{(\Omega S)}\left(\frac{a}{H}\right)^{5}\right] .
\end{aligned}
$$

Finally, for the coupling between rate of strain and stresslet, the mobility tensor is 


$$
\begin{aligned}
\mathbf{M}_{E S}= & \frac{1}{6 \pi \eta a^{3}}\left\{[ - \frac { 9 } { 3 0 } - f _ { 3 } ^ { ( E S ) } ( \Xi ) ( \frac { a } { H } ) ^ { 3 } + f _ { 5 } ^ { ( E S ) } ( \Xi ) ( \frac { a } { H } ) ^ { 5 } - f _ { 7 } ^ { ( E S ) } ( \Xi ) ( \frac { a } { H } ) ^ { 7 } ] \left[\left(\delta_{i j}-\delta_{i 3} \delta_{j 3}\right)\left(\delta_{k l}-\delta_{k 3} \delta_{l 3}\right)\right.\right. \\
& \left.-2\left(\delta_{i j}-\delta_{i 3} \delta_{j 3}\right) \delta_{k 3} \delta_{l 3}-2 \delta_{i 3} \delta_{j 3}\left(\delta_{k l}-\delta_{k 3} \delta_{l 3}\right)+4 \delta_{i 3} \delta_{j 3} \delta_{k 3} \delta_{l 3}\right] \\
& +\left[\frac{9}{20}-g_{3}^{(E S)}(\Xi)\left(\frac{a}{H}\right)^{3}+g_{5}^{(E S)}(\Xi)\left(\frac{a}{H}\right)^{5}-g_{7}^{(E S)}(\Xi)\left(\frac{a}{H}\right)^{7}\right] \times\left[\left(\delta_{i k}-\delta_{i 3} \delta_{k 3}\right)\left(\delta_{j l}-\delta_{j 3} \delta_{l 3}\right)+\left(\delta_{i l}-\delta_{i 3} \delta_{l 3}\right)\left(\delta_{j k}-\delta_{j 3} \delta_{k 3}\right)\right. \\
& \left.-2\left(\delta_{i j}-\delta_{i 3} \delta_{j 3}\right) \delta_{k 3} \delta_{l 3}-2 \delta_{i 3} \delta_{j 3}\left(\delta_{k l}-\delta_{k 3} \delta_{l 3}\right)+4 \delta_{i 3} \delta_{j 3} \delta_{k 3} \delta_{l 3}\right]+\left[\frac{9}{20}-h_{3}^{(E S)}(\Xi)\left(\frac{a}{H}\right)^{3}+h_{5}^{(E S)}(\Xi)\left(\frac{a}{H}\right)^{5}-h_{7}^{(E S)}(\Xi)\right. \\
& \left.\left.\times\left(\frac{a}{H}\right)^{7}\right] \times\left[\left(\delta_{i k}-\delta_{i 3} \delta_{k 3}\right) \delta_{j 3} \delta_{l 3}+\left(\delta_{i l}-\delta_{i 3} \delta_{l 3}\right) \delta_{j 3} \delta_{k 3}+\left(\delta_{j k}-\delta_{j 3} \delta_{k 3}\right) \delta_{i 3} \delta_{l 3}+\left(\delta_{j l}-\delta_{j 3} \delta_{l 3}\right) \delta_{i 3} \delta_{k 3}\right]\right\} \boldsymbol{\delta}_{i} \boldsymbol{\delta}_{j} \boldsymbol{\delta}_{k} \boldsymbol{\delta}_{l} .
\end{aligned}
$$

Other higher order mobility tensors can be calculated in a similar fashion, but for the purposes of dynamic simulation it has proven convenient to truncate the force multipoles at the stresslet level. Therefore, we omit the calculation of any higher order terms. It is true that just as in calculations involving many particles, when the particle is close to one of the channel walls, all the force multipoles are important since this constitutes the lubrication limit. We use the Stokesian dynamics method of constructing the grand mobility tensor and its inverse, the grand resistance tensor, to resolve this difficulty.

\section{Stokesian dynamics}

The Stokesian dynamics method simplifies the calculation of hydrodynamic interactions among many bodies in Stokes flow by first computing the correct pair-wise, longrange behavior of the particles in the form of the grand mobility tensor. This is analogous to what we have done in Secs. II A-II C. Durlofsky, Brady, and Bossis ${ }^{26}$ showed that inverting the grand mobility tensor is equivalent to a method of reflections type procedure that yields the many-body longrange interactions among the particles. Since the grand mobility tensor must in every practical sense be truncated at some force multipole level, this invert alone fails to account for any pair-wise lubrication interactions which may occur when surfaces are nearly touching. The truncated invert is typically designated as $\mathcal{R}^{\infty}$ and is a far-field contribution to the grand resistance tensor which is denoted more generally as

$$
\mathcal{R}=\left(\begin{array}{cccc}
\mathbf{R}_{F U} & \mathbf{R}_{F \Omega} & \mathbf{R}_{F E} & \ldots \\
\mathbf{R}_{L U} & \mathbf{R}_{L \Omega} & \mathbf{R}_{L E} & \ldots \\
\mathbf{R}_{S U} & \mathbf{R}_{S \Omega} & \mathbf{R}_{S E} & \ldots \\
\vdots & \vdots & \vdots & \ddots
\end{array}\right),
$$

where R's are the individual resistance tensors. The shortcomings of the far-field resistance tensor are overcome by writing the grand resistance tensor as

$$
\mathcal{R}=\mathcal{R}^{\infty}+\mathcal{R}^{2 B}-\mathcal{R}^{2 B, \infty},
$$

where $\mathcal{R}^{2 B}$ is the exact pair-wise lubrication form of the grand resistance tensor and $\mathcal{R}^{2 B, \infty}$ is the two body far-field form of the grand resistance tensor. In this way, our approximation for the grand resistance tensor accounts for the correct many bodied far-field interactions and the correct pairwise lubrication interactions. We can include the walls in these calculations by simply adding in the correct lubrication interactions of each particle with each wall individually and subtracting out the far-field interactions with that same wall as though it were in an otherwise unbounded domain. This is represented schematically as

$$
\mathcal{R}=\mathcal{R}^{\infty}+\mathcal{R}^{2 B}-\mathcal{R}^{2 B, \infty}+\mathcal{R}^{W}-\mathcal{R}^{W, \infty},
$$

where $\mathcal{R}^{W}$ and $\mathcal{R}^{W, \infty}$ are the exact lubrication and far-field resistance tensors for interactions with each wall individually. Bossis, Meunier, and Sherwood ${ }^{27}$ tabulated the lubrication expressions to the stresslet level of truncation, and Swan and Brady ${ }^{4}$ computed the far-field, single wall interactions to that same level.

The dynamics of particles are straightforward to work out once the grand resistance tensor is known. Newtonian mechanics state that the particle's acceleration must balance with the forces applied to it, viz.,

$$
\frac{\partial}{\partial t} \mathbf{m} \cdot \mathbf{U}=\mathbf{F}^{H}+\mathbf{F}^{P}
$$

where $\mathbf{m}$ is the generalized mass tensor for all the particles and $\mathbf{F}^{P}$ is any additional force on the particles. This might include Brownian forces, gravity, or forces due to an electric field, among others. For small particles with a density $\left(\rho_{p}\right)$ not too dissimilar from that of the fluid, the acceleration of the particles is not important. That is, when the Stokes number, $\rho_{p} U a / \eta$, which measures the relative importance of particle inertia, is small, the left hand side of the particle equations of motion is made identically equal to zero. In this way, Eq. (44) becomes

$$
\frac{\partial \mathbf{X}}{\partial t}=\mathbf{U}^{\infty}+\mathbf{R}_{F U}^{-1} \cdot \mathbf{F}^{P},
$$

where $\mathbf{X}$ is the position of the particles whose time derivative is simply $\mathbf{U}$. Integration of this equation is not too complicated but the focus of this article is the calculation of $\mathbf{R}_{F U}$, 
etc. The work by Phung, Brady, and Bossis ${ }^{28}$ offers a broad overview of how this may be accomplished. This schematic makes it possible to compute all of the hydrodynamic and rheological results presented in Sec. III.

\section{RESULTS AND DISCUSSIONS}

\section{A. Components of the single particle mobility in a channel}

In this section we plot and discuss the functions of the fractional channel distance, $\Xi$, which in essence define the different elements of the grand mobility tensor. The functions we plot here are perhaps most useful in a tabulated form for many values of $\Xi$ distributed within $(0,1)$ —although such tables are difficult to represent in print. They are, however, available from the editorial office. To begin, consider the plots of $f_{1}^{(U F)}(\Xi), f_{3}^{(U F)}(\Xi)$, and $f_{5}^{(U F)}(\Xi)$ shown in Fig. 2. Faxén computed the force $F$ on a particle translating parallel to the walls with a velocity $U$, halfway and a quarter of the way across a channel. His results were presented as a ratio of the velocity to the force, viz.,

$$
\frac{6 \pi \eta a U}{F}=\left\{\begin{array}{cc}
1-0.6526\left(\frac{a}{h}\right)+0.1475\left(\frac{a}{h}\right)^{3}-0.131\left(\frac{a}{h}\right)^{4}-0.0644\left(\frac{a}{h}\right)^{5}+\cdots, & h=\frac{H}{4} \\
1-1.004\left(\frac{a}{h}\right)+0.418\left(\frac{a}{h}\right)^{3}+0.21\left(\frac{a}{h}\right)^{4}-0.169\left(\frac{a}{h}\right)^{5}+\cdots, & h=\frac{H}{2}
\end{array}\right.
$$

First, notice that his expression is equivalent to the inverse of the resistance tensor $\mathbf{R}_{F U}$ and is closely related to $\mathbf{M}_{U F}$. Where $\mathbf{R}_{F U}^{-1}$ is an infinite series in powers of $a / h$, it is equivalent to leading order to $\mathbf{M}_{U F}$, which can be represented with a finite number of terms. The additional $(a / h)^{4}$ term as well as the rest of the series represented by the ellipses in Faxén's expressions is due to higher order force moments. In fact, we find that the coefficients Faxén computed are identical to our results when rescaled so that his expressions are written in terms of inverse powers of $H$ instead of inverse powers of $h$. One result Faxén opted not to generate was the mobility for a particle sedimenting perpendicular to the channel walls. However, we have computed these mobility terms and find a behavior similar to the sedimentation parallel to the wall. Interesting, although perhaps not surprising, is the fact that sedimentation toward the wall is always slower than sedimentation along the wall. This must be because it is always harder to squeeze fluid out of a gap than to just push it aside at low Reynolds number.

It might be disconcerting to see the mobility coefficients diverge as $\Xi$ approaches zero and unity. However, this behavior is correct as it leads to the conclusion that the parallel wall problem collapses to the single wall problem in the limit that the channel spacing is infinitely wide. Suppose the particle is fixed against the wall such that $\Xi=a / H$. This is the closest the particle will ever get to wall regardless of the value of $H$ and therefore, this is the smallest value of $\Xi$ possible. We take the limit of the mobility tensor as $a / H$ goes to zero and discover a satisfying, finite result. To simplify things, we examine the limits of each contribution to the mobility tensor individually,

$$
\begin{array}{ll}
\lim _{\Xi \rightarrow 0} f_{1}^{(U F)}(\Xi) \Xi=\frac{9}{16}, & \lim _{\Xi \rightarrow 0} g_{1}^{(U F)}(\Xi) \Xi=\frac{9}{8}, \\
\lim _{\Xi \rightarrow 0} f_{3}^{(U F)}(\Xi) \Xi^{3}=\frac{1}{8}, & \lim _{\Xi \rightarrow 0} g_{3}^{(U F)}(\Xi) \Xi^{3}=\frac{1}{2},
\end{array}
$$

$$
\lim _{\Xi \rightarrow 0} f_{5}^{(U F)}(\Xi) \Xi^{5}=\frac{1}{16}, \quad \lim _{\Xi \rightarrow 0} f_{5}^{(U F)}(\Xi) \Xi^{5}=\frac{1}{8} .
$$

These results are easily recognized as the coefficients of the single wall mobility tensor, ${ }^{4}$ which is sometimes written as

$$
\begin{aligned}
\mathbf{M}_{U F}= & \frac{1}{6 \pi \eta a}\left\{\left(\mathbf{I}-\boldsymbol{\delta}_{3} \boldsymbol{\delta}_{3}\right)\left[1-\frac{9}{16}\left(\frac{a}{h}\right)+\frac{1}{8}\left(\frac{a}{h}\right)^{3}-\frac{1}{16}\left(\frac{a}{h}\right)^{5}\right]\right. \\
& \left.+\boldsymbol{\delta}_{3} \boldsymbol{\delta}_{3}\left[1-\frac{9}{8}\left(\frac{a}{h}\right)+\frac{1}{2}\left(\frac{a}{h}\right)^{3}-\frac{1}{8}\left(\frac{a}{h}\right)^{5}\right]\right\},
\end{aligned}
$$

where $h$ is the height of the particle above that single wall. In the above limit, $a / h$ is clearly unity, and we recognize that, indeed, the two wall problem collapses into the single wall problem when the channel width tends to infinity. This also makes it clear that the asymptotic behavior as $\Xi \rightarrow 0$ of the mobility coefficients must be

$$
f_{i}^{(U F)}(\Xi), \quad g_{i}^{(U F)}(\Xi) \sim \frac{1}{\Xi^{i}},
$$

where $i=1,3,5$ and the coefficient of proportionality is given by the single wall value. This result is particularly useful in the computational domain since tabulation cannot be performed for all values of $\Xi$, and in the quickly varying regions near the walls the asymptotic result can be used as a substitute for the tabulated one.

For the coupling between translation and torque, we find a similar set of expressions. These are more challenging to visualize since they switch sign as the particle moves across the channel. This is easy to understand by considering the walls individually. For a particle above a single wall, the torque also couples to the translation of the particle. Flipping the coordinate system over so that the wall is now above the particle while keeping the torque the same results in translation in the opposite direction; hence the change in sign. The components of $\mathbf{M}_{U L}$ are plotted in Fig. 3. One interesting 

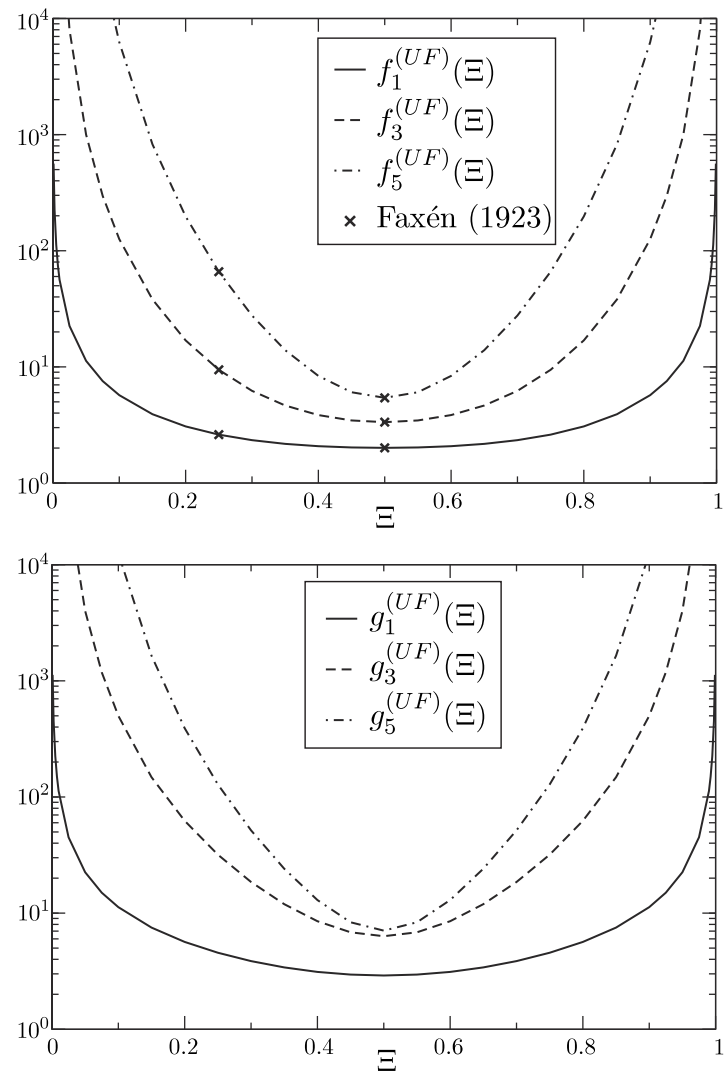

FIG. 2. The components of the translation-force coupling in the directions parallel and perpendicular to the walls, respectively.

feature of this particular coupling is the $O(a / H)^{2}$ contribution. There is no analogous contribution in the single wall problem. Near the middle of a relatively large channel, this contribution dominates both the translation-torque and the rotation-force couplings. Although unexpected, this result is consistent with the single wall results as $f_{2}^{(U L)}(\Xi)$ is nonsingular near the walls and therefore makes no contribution when only one wall is present. This term represents a lower order interaction generated by reflecting a rotlet between a pair of walls. In the single wall case where the domain is unbounded, there is only one no-slip condition to enforce and the parts of the disturbance velocity leading to this contribution are identically zero in order for the magnitude of the reflected velocity to remain finite in the far-field. An analogous set of plots shown in Fig. 4 has been generated for the translation-stresslet coupling. The sign of the mobility coefficients also changes as the particle moves from one wall to another; therefore, only the domain $\Xi \in(0,0.5)$ is depicted. This is most easily understood by recognizing that while $\mathbf{U}$ and $\mathbf{S}$ are preserved under the coordinate transformation that swaps the lower and upper walls, the tensor coupling these two is third order and depends on an odd number of tensor products of the unit vector $\boldsymbol{\delta}_{3}$. When the coordinate system flips, so does the sign of the unit vector and the coupling functions react accordingly to preserve the sign of the expression. Because of the symmetry between the walls, the coupling between translation and stresslet, as well as translation and torque, must go to zero when the particle is
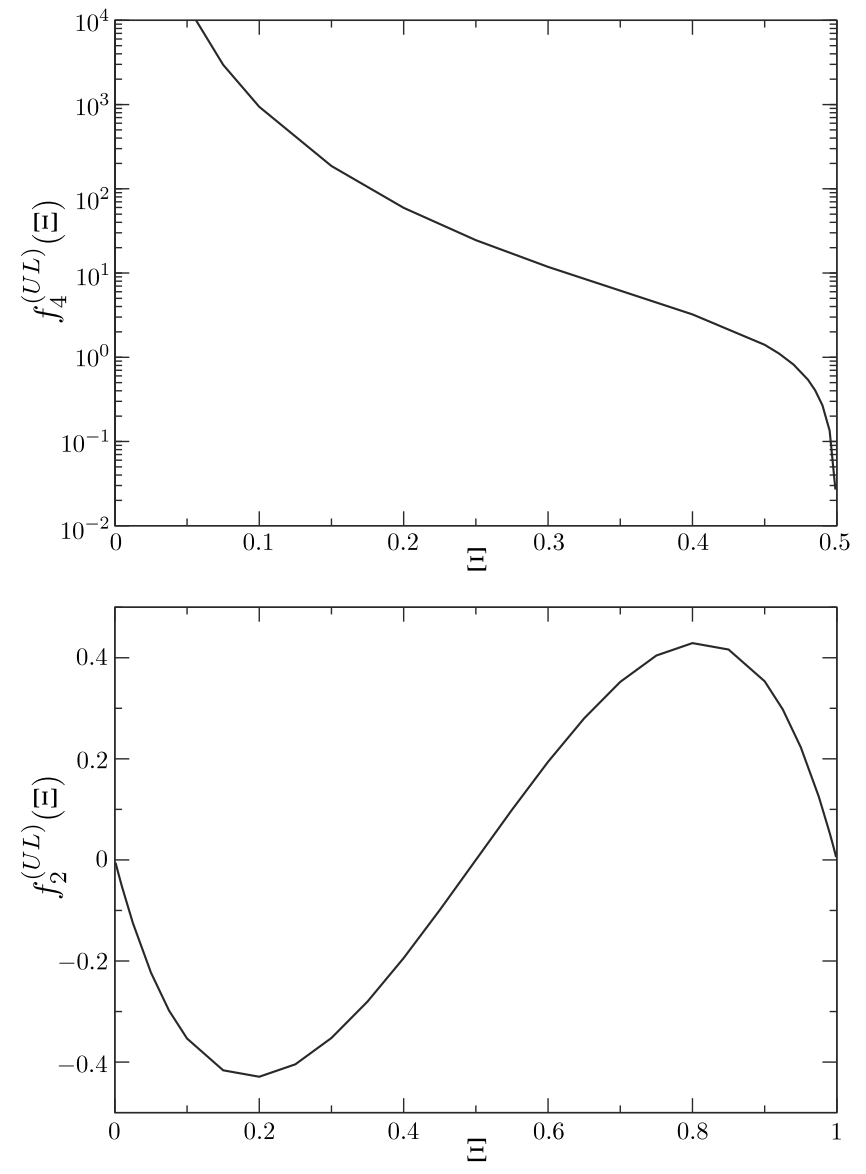

FIG. 3. The components of the translation-torque coupling. The $O(a / H)^{2}$ contribution is not singular and therefore makes no contribution to the single wall problem.

halfway across the channel. Additionally, the singular limits of each coefficient approach the corresponding single wall values.

We plot the coefficients associated with the rotationtorque coupling in Fig. 5. As with the mobility of a particle above a single wall, the no-slip condition on the boundaries retards rotation about the axes parallel to the walls more than rotation about the axis perpendicular to the walls. These coefficients are symmetric across the channel because under the coordinate inversion which switches the walls, the rotation and torque, which are handed quantities, both change sign. Similarly, the coupling between rotation and stresslet is also symmetric across the channel. These coefficients are plotted in Fig. 6. These also approach the single wall values in the limit that $\Xi$ approaches zero and unity.

The coupling between rate of strain and stresslet is key for computing the shear stress in a force- and torque-free suspension. For a dilute suspension, the average of this coupling across the channel is precisely the particle contribution to the shear stress. Each of the coefficients of this coupling is plotted in Fig. 7.

The single wall results are recovered as the particle approaches each wall. Interestingly, the magnitude of these coefficients is approximately a factor of 10 larger than all the others presented. Relative to the scale factors $(a / H)^{n}$, the coupling between rate of strain and stresslet is quite strong 

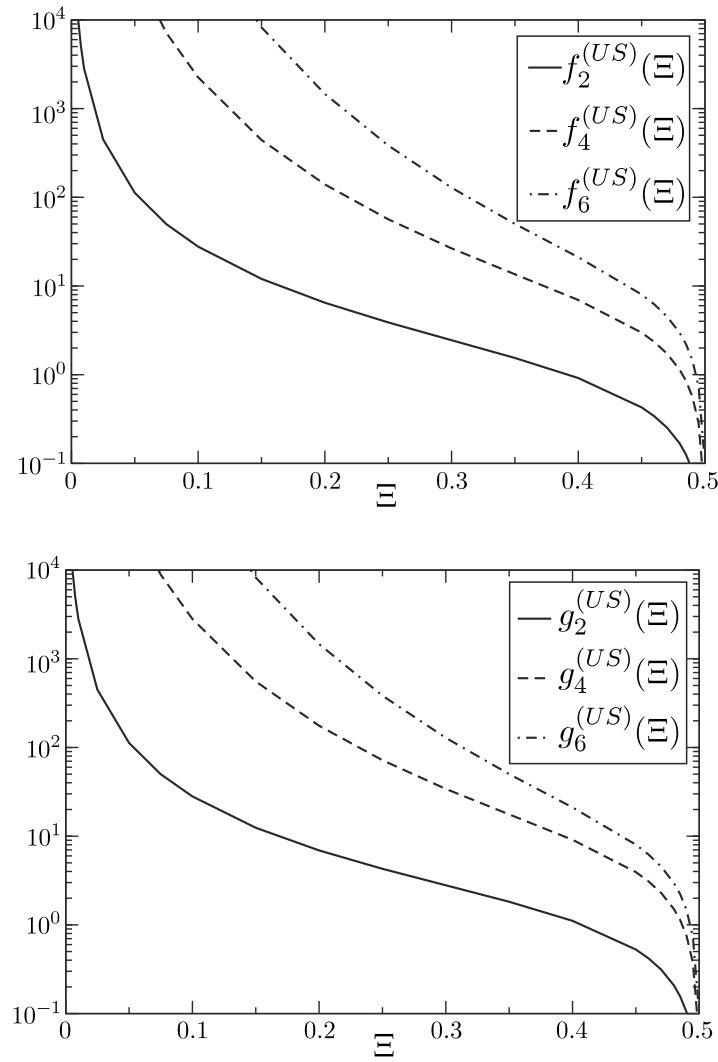

FIG. 4. The components of the translation-stresslet coupling corresponding to couples between translation parallel to the walls and the stresslet $S_{33}$ and translation perpendicular to the walls and stresslets with components parallel to the walls as well as translation perpendicular to the wall and the stresslet $S_{33}$ via superposition.

when compared to the other equivalently scaled coefficients (i.e., $\mathbf{M}_{\Omega L}, \mathbf{M}_{\Omega S}$ ). Therefore, the walls should have a significant impact on the measurement of the shear viscosity of a dilute suspension in a channel.

We conclude this section by examining Oseen's superposition approximation for the contribution to the mobility between a pair of walls. For the translation-force coupling terms $f_{1}^{(U F)}(\Xi)$ and $g_{1}^{(U F)}(\Xi)$, Oseen's superposition approximation would generate the approximate coefficients,

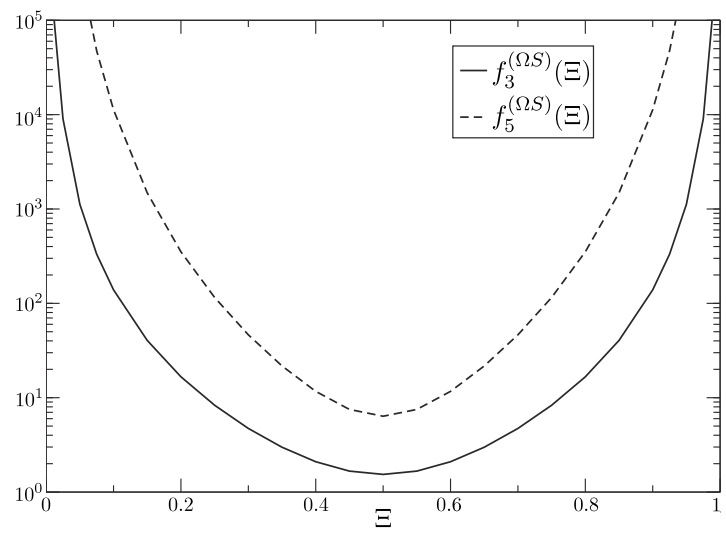

FIG. 5. The components of the rotation-torque coupling about the axes parallel and perpendicular to the walls, respectively.

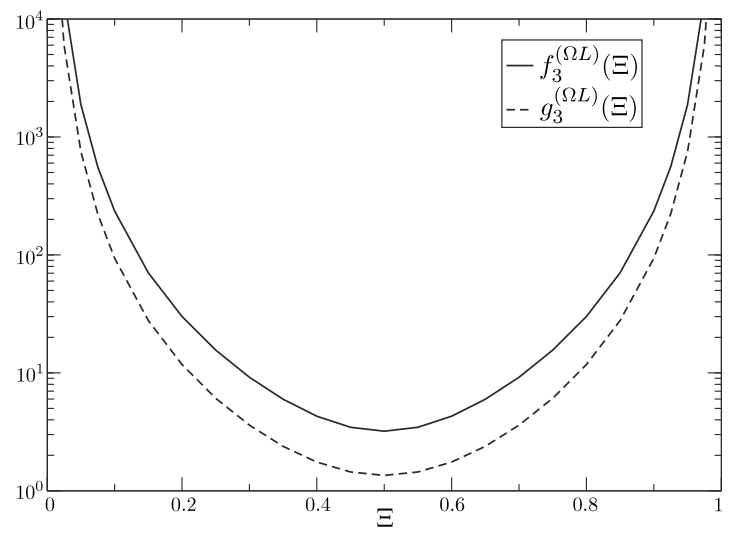

FIG. 6. The components of the rotation-stresslet coupling which relates rotation of a particle about the axes parallel to the walls to the stresslet.

$$
\begin{aligned}
& \bar{f}_{1}^{(U F)}(\Xi)=\frac{9}{16}\left(\frac{1}{\Xi}+\frac{1}{1-\Xi}\right), \\
& \bar{g}_{1}^{(U F)}(\Xi)=\frac{9}{8}\left(\frac{1}{\Xi}+\frac{1}{1-\Xi}\right) .
\end{aligned}
$$

We plot these in Fig. 8 along with $f_{1}^{(U F)}(\Xi)$ and $g_{1}^{(U F)}(\Xi)$. The curves are qualitatively similar. Of course, since data on logarithmic axes can often appear quite close, we also compute the relative error between the exact two wall results and the superposition approximation. Near each of the walls, the superposition approximation performs quite well with less than $5 \%$ error when the particle is less than $5 \%$ of the way across the channel. However, in the middle of the channel, the error balloons quickly. In the case of translation perpendicular to the walls, the error is nearly $60 \%$ in the middle of the channel. This is likely because the walls "leak" fluid and allow for "slip" in the superposition approximation as they do not satisfy the no-slip condition. That is, the singularity solution for a single wall satisfies the no-slip condition on that wall alone while allowing free motion of the fluid everywhere else in the superior half-space. The fluid continues to flow freely in this space even when the singularity solution for the second channel wall is introduced. Therefore, the condition that the velocity of the fluid is equivalent to the velocity of the wall at contact can never be satisfied. When the source of the flow originates near one of the walls, the resulting flow is relatively weak, however. Consequently, the error in the superposition approximation is small near the wall. As little fluid is set into motion in these cases, we can confidently employ the Stokesian dynamics method of adding in the lubrication forces while subtracting the single wall forces from the problem in all but the most narrow channels. Even in narrow channels, the effective superposition of resistance tensors may still be quite good, as inverting the grand mobility tensor accounts for all the reflections between the particle and the walls. The biggest contribution to this is simply the single wall portion when $\Xi$ is near zero or unity, so removing this portion directly should be quite effective. Determining the exact error associated with this approximation is difficult in general, but a similar approximation is used to add in the pair-wise lubrication interactions among 

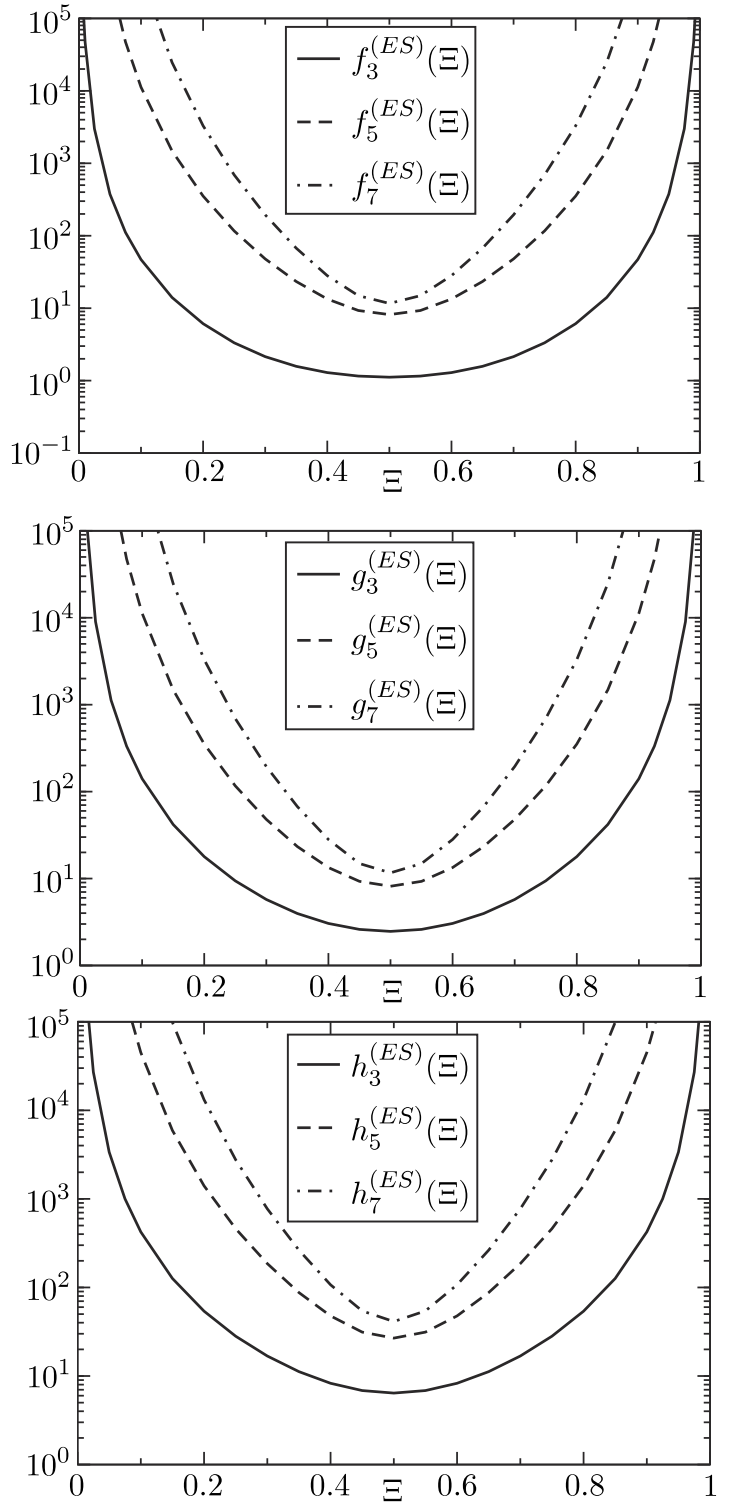

FIG. 7. The components of the rate of strain-stresslet coupling. Between two walls, there are only three independent components of the tensor $\mathbf{M}_{E S}$ corresponding to the necessary Stokes flow symmetries and the anisotropy caused by the wall.

many particles and has proven very effective for determining accurate and consistent rheological data. ${ }^{28}$

\section{B. Sedimentation of a particle between parallel walls}

We use a Stokesian dynamics simulation to compute the velocity of a particle falling due to gravity between parallel walls. In Fig. 9, we plot the parallel fall speed and rotation rate of a single particle in channels of various widths. In order to compare the results for different channel widths on the same basis, we plot them as a function of the height of the particle above the lower wall in the channel divided by the channel width (i.e., $\Xi-a / H$ ). Because of the symmetry of the channel, we only consider particles which sit less than halfway across the channel. In this way, we capture both the lubrication and midchannel behavior of the fall speed and the rotation rate graphically.
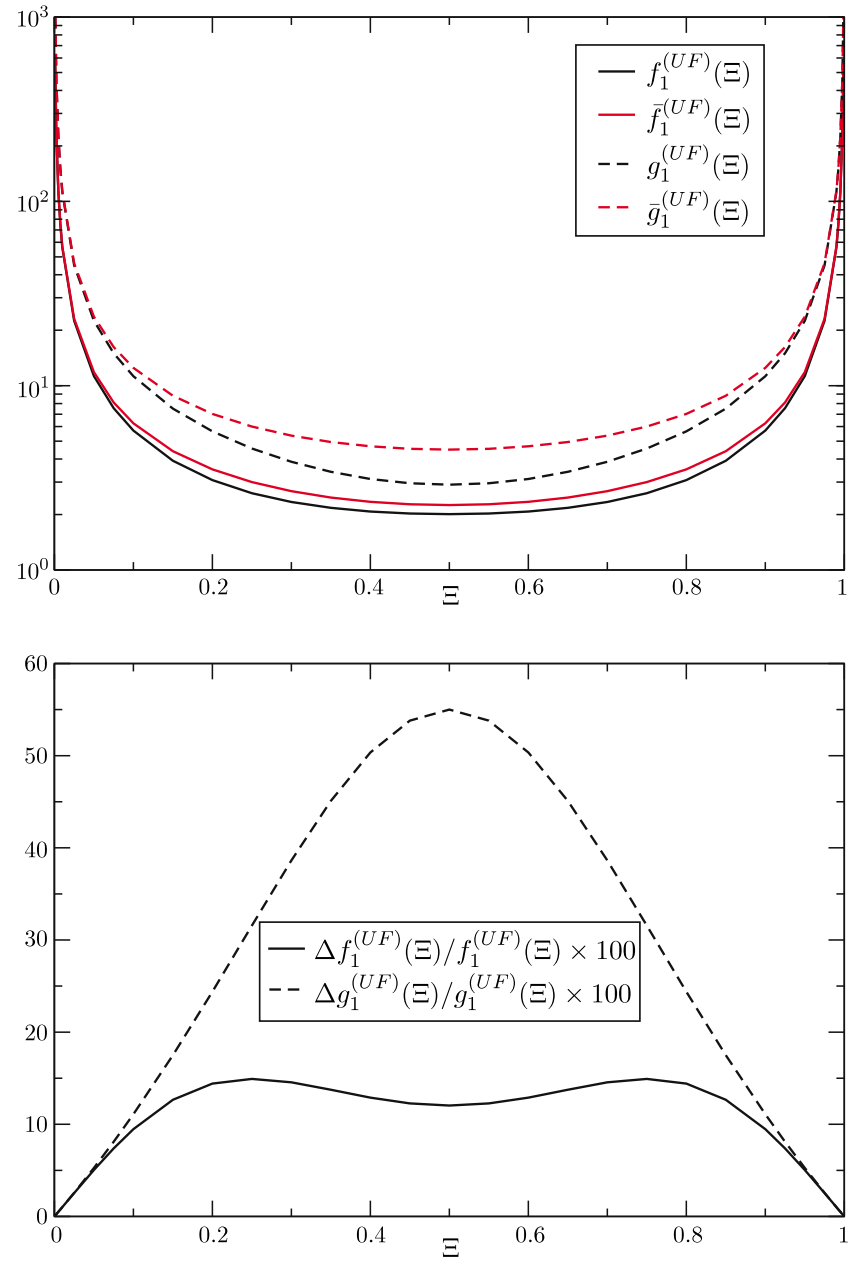

FIG. 8. (Color online) The components of the exact translation-force coupling and the translation-force coupling determined using Oseen's superposition approximation as well as the relative error between this and the Stokesian dynamics results.

Notice that because of the interactions between the walls, the midchannel velocity is a function of the channel width. For sufficiently wide channels $(H / a \gg 1)$, the fall speed in the middle of the channel approaches unbounded value $\left(6 \pi \eta a F_{\|}\right)$as $a / H$. This result arises directly from the expression for $\mathbf{M}_{U F}$ constructed in the previous section. Similarly, for wide channels, the $O(a / H)^{2}$ contribution to the rotation-force coupling is explicitly observable. In the near wall region, the lubrication forces cause the particle to rotate one direction, like a wheel rolling over the ground. However, near the middle of the channel, this rotation reverses briefly because the $O(a / H)^{2}$ term dominates the interaction. Remember, this term only arises because of the presence of both walls. It has no analog in the single wall problem. While this reversal may seem anomalous, we can refer back to Faxén's result for the force on a particle falling in a channel to confirm that this contribution makes a substantial contribution to the dynamics of the particle. In Faxén's solution, there was an $O(a / H)^{4}$ contribution to the fall speed of a particle in a channel. Of course, that problem studied a particle which did not rotate as it fell, so the proper mobility must be written as 

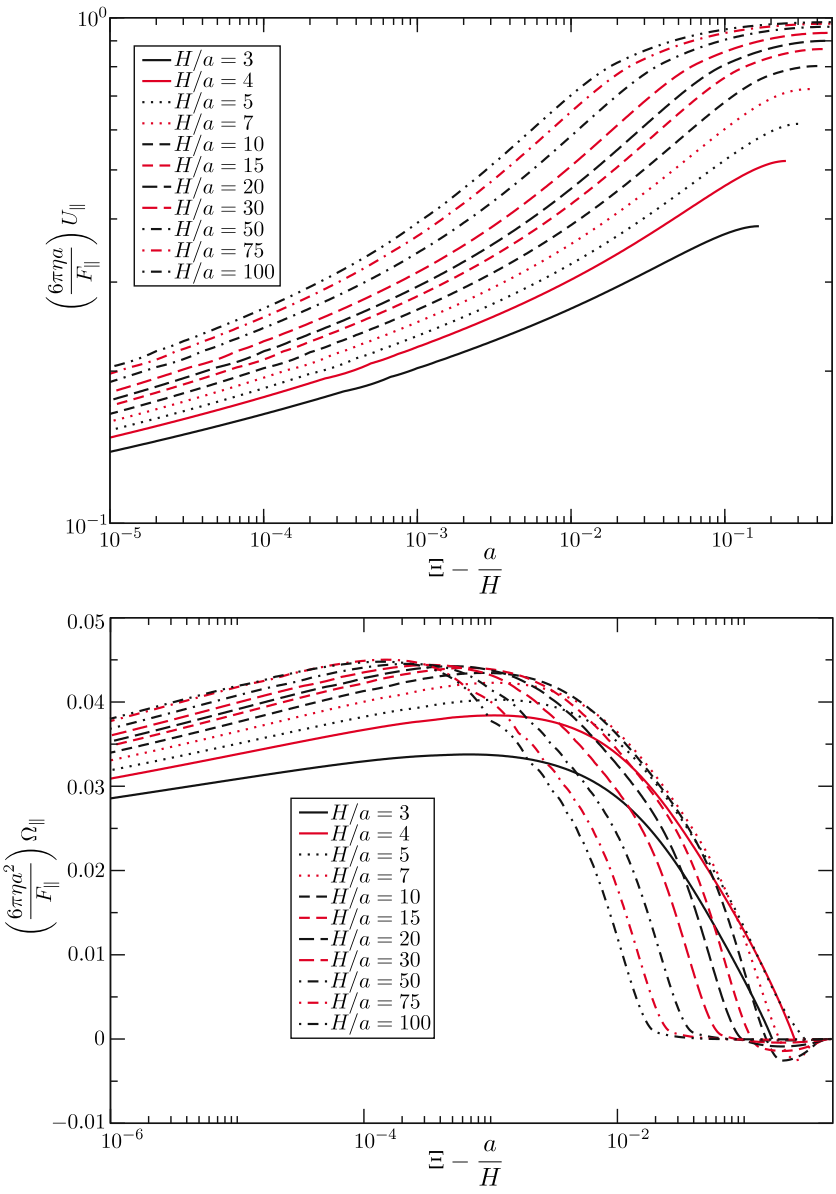

FIG. 9. (Color online) The fall speed, $U_{\|}$, and rotation rate, $\Omega_{\|}$, of a particle sedimenting along a channel. The fall speed and rotation rate are normalized by the Stokes velocity of the same particle subject to the same force in an otherwise unbounded fluid (i.e., $F_{\|} / 6 \pi \eta a$ and $F_{\|} / 6 \pi \eta a^{2}$ ).

$$
\mathbf{M}_{U F}-\mathbf{M}_{U L} \cdot \mathbf{M}_{\Omega L}^{-1} \cdot \mathbf{M}_{\Omega F} .
$$

We have shown that we recover Faxén's $O(a / H), O(a / H)^{3}$, and $O(a / H)^{5}$ terms in $\mathbf{M}_{U F}$. Recall now that to leading order, $\mathbf{M}_{U L}$ and $\mathbf{M}_{\Omega F}$ scale like $O(a / H)^{2}$ and $\mathbf{M}_{\Omega L}$ is simply $O(1)$. From this, it is clear that the additional $O(a / H)^{4}$ piece of Faxén's result is due in part to the two wall reflection of the rotlet - the remainder is due to the induced stresslet. This rotational reversal may be difficult to observe experimentally as it happens only over a narrow band near the center of the channel where the rotation rate of the particle is already quite small. However, given the precise control possible with laser tweezer techniques, it seems likely that this could be measured in a particle tracking experiment.

One must be careful when making such a comparison, however, as we have neglected one formality in our analysis. In addition to the boundary conditions specified at the channel walls, a condition on the mean flow through or the pressure drop down the channel is necessary. These represent closed and open channel ends. Implicit in what we have calculated is the restriction that the pressure drop down the channel is zero. Since the space between the channel walls is infinite, there are no backflow effects hindering the motion of the particle. The same may not be true in the finite volume of an experimental apparatus.

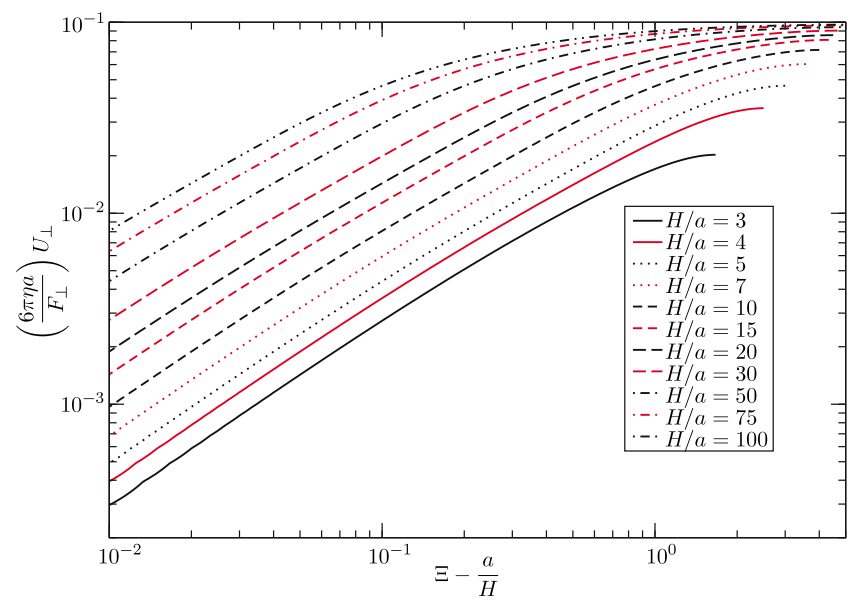

FIG. 10. (Color online) The fall speed, $U_{\perp}$, of a particle sedimenting along a channel normalized by the Stokes velocity of the same particle subject to the same force in an otherwise unbounded fluid (i.e., $F_{\perp} / 6 \pi \eta a$ ).

We also plot the fall speed of a particle sedimenting perpendicular to the channel walls as a function of position in the channel in Fig. 10. By symmetry, there can be no rotational coupling in this case. Qualitatively, this figure is quite similar to that for sedimentation along the channel. However, one distinct difference is the decay rate of the fall speed in the near wall regime. Motion toward the wall is much more resistive in this regime since the lubrication forces scale like $(\Xi-a / H)^{-1}$. For motion along the wall, the lubrication forces are more weakly singular and scale logarithmically. Of particular interest is the fraction of the channel over which the sedimentation velocity is near the midchannel velocity. In other words, we would like to know which region of the channel is least sensitive to the presence of the walls. To measure this, we choose an arbitrary threshold of $5 \%$ of the midchannel velocity and measure the fractional distance across the channel where a particle first obtains this fall speed. We use this to calculate the fraction of the channel over which the mean fall speed of the particle is greater than $95 \%$ of the midchannel fall speed. This fraction is plotted against the channel width in Fig. 11. We expect

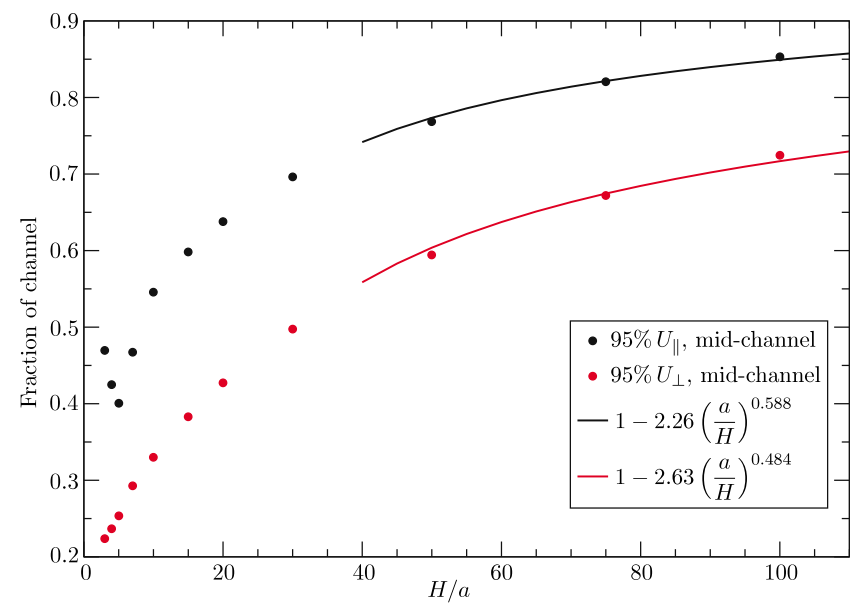

FIG. 11. (Color online) The fraction of the channel over which a particle sediments at $95 \%$ of its midchannel fall speed. 
that as the channel becomes wider, the fraction of the channel where sedimentation is near the midchannel speed will grow monotonically. There is the possibility that the Stokesian dynamics model of the near wall interactions "overcounts" the resistivity of each of the walls when the separation is small, however. While the lubrication interactions each scale singularly with respect to the gap width between the particle and the wall and are strictly independent, the presence of a nearby second wall affects the $O(1)$ contribution to the resistance. Our approach recovers the dominant hydrodynamic effect via these singular contributions. For channels outside the narrow regime (i.e., $H / a>20$ ), we find that the fraction of the channel where the particle falls faster than $95 \%$ of the midchannel speed scales roughly as the inverse square root of the channel width. Qualitatively, the fast-fall-speed fraction of channel grows in a way which is independent of the fall direction. While the absolute channel fraction for perpendicular sedimentation is always less than parallel sedimentation, the growth rate is comparable. As the channel gets very large $(H / a \gg 1)$, the difference between parallel and perpendicular sedimentation becomes negligible everywhere but near the walls. We expect that these two curves coincide in the limit that the gap between the walls is infinitely wide.

\section{Brownian drift of a particle in a channel}

The stochastic thermal forces on a particle in a channel lead to a deterministic contribution to the particle's velocity arising from the dependence of the Brownian forces on the hydrodynamic interactions of the particle with the channel walls. Given a particle near a wall at a particular instant in time, thermal forces will drive it either toward or away from the wall with equal probability. If the particle moves toward the wall, its mobility decreases and the thermal impulse propels the particle more slowly. Conversely, if the particle moves away from the wall, the impulse propels the particle more quickly. Of course the strength of the thermal forcing varies with distance from the wall as well since it is proportional to the square root of the hydrodynamic resistance. Substituting an instantaneously correlated thermal impulse denoted $\mathbf{F}^{B}(t)$ of zero mean and rms strength $\sqrt{2 k T \mathbf{R}_{F U}[\mathbf{X}(t)]}$ into Eq. (45), integrating over an interval in time $(\Delta t)$ that is short with respect to the particle diffusion time and taking care to account for changes in the resistance as the particle diffuses yields an evolution equation for the position of the particle,

$$
\begin{aligned}
\mathbf{X}(t+\Delta t)= & \mathbf{X}(t)+\mathbf{R}_{F U}^{-1}[\mathbf{X}(t)] \cdot \mathbf{F}^{B}(t) \sqrt{\Delta t} \\
& +k T \nabla \cdot \mathbf{R}_{F U}^{-1}[\mathbf{X}(t)] \Delta t+O\left(\Delta t^{2}\right) .
\end{aligned}
$$

The mean effect of the change in the hydrodynamic resistance and in the magnitude of the thermal forcing as a particle diffuses in space results in a deterministic drift away from the wall. The drift velocity is given quite simply by $k T \nabla \cdot \mathbf{R}_{F U}^{-1}$, where $k T$ is the thermal energy. ${ }^{29,30}$ There is no drift parallel to the wall because the resistance is independent of the position along the wall. In Fig. 12 we plot the Brownian drift velocity of a particle sitting above the lower wall for

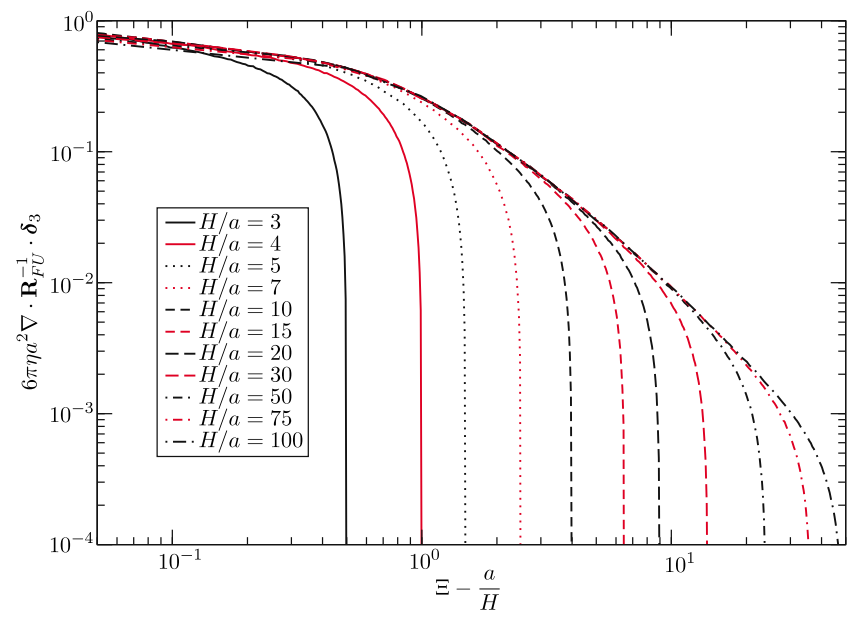

FIG. 12. (Color online) The drift velocity of a single Brownian particle in channel of width $\mathrm{H} / \mathrm{a}$ plotted as a function of height above the lower channel wall.

channels of various widths. As the walls are further separated, the particle's sense of the second wall becomes minimal and the drift velocity decays at the single wall rate as $h^{-2}$. However, the drift velocity decays to zero quickly as the particle approaches the midline of the channel where there is no variation in mobility. On the other side of the midline, the drift reverses sign as the particle is impelled away from the upper wall instead.

To highlight the importance of the Brownian drift, consider a single particle in a channel with hard walls. The equilibrium distribution for the particle's position in the channel is given by the Boltzmann distribution and is therefore uniform across the channel. Now, place the particle arbitrarily close to one of the walls. Because the hydrodynamic resistance is large, the diffusivity of the particle is practically zero. If we allow the particle to move according to Eq. (55) but neglect the contribution due to Brownian drift, then particle hardly moves, and the probability of finding the particle at this initial location is unity for all time. The equilibrium, uniform distribution is never recovered, although it must be. The inclusion of the Brownian drift drives the particle away from the wall deterministically by exactly the amount required such that the probability of finding the particle anywhere in the channel at long times is uniform. In fact, that is the function of the Brownian drift. It is an entropic/thermal/ stochastic mechanism that drives a stationary Markov process toward the stationary solution.

\section{Einstein viscosity for a dilute suspension between parallel walls}

We begin this calculation with Brenner's definition of the viscosity as the coefficient of proportionality relating energy dissipation in two similarly strained flows, viz.,

$$
\frac{E^{(0)}}{\eta}=\frac{E^{(0)}+E^{*}}{\eta^{*}}
$$

where $E^{(0)}$ is the rate of energy dissipation in a particle free flow, $E^{*}$ is the additional rate of energy dissipation in a particle laden flow with the same rate of strain and solvent vis- 
cosity, and $\eta^{*}$ is the viscosity of that suspension. ${ }^{12}$ With some relatively simple manipulation, one can show that

$$
\frac{\eta^{*}}{\eta}=1-\varphi \frac{\langle\mathbf{S}\rangle: \mathbf{E}^{\infty}}{\frac{8}{3} \pi \eta a^{3} \mathbf{E}^{\infty}: \mathbf{E}^{\infty}},
$$

where $\langle\mathbf{S}\rangle$ is the average particle stresslet and $\varphi$ is the particle volume fraction. For a force and torque free suspension, we conclude that the particle stresslet is simply

$$
\mathbf{S} \approx-\mathbf{M}_{E S}^{-1}: \mathbf{E}^{\infty},
$$

where the approximation is valid when the particles are far from the walls such that lubrication forces are unimportant. We will use this approximation throughout the rest of this section as we are interested in the viscosity of dilute suspensions in channels which are wide. In this case, a small fraction of the particles are found near the walls and therefore, any lubrication effects make a minimal contribution to the rate of energy dissipation. With this simplification, our expression for the viscosity of the suspension becomes

$$
\frac{\eta^{*}}{\eta}=1+\varphi \frac{\mathbf{E}^{\infty}:\left\langle\mathbf{M}_{E S}^{-1}\right\rangle: \mathbf{E}^{\infty}}{\frac{8}{3} \pi \eta a^{3} \mathbf{E}^{\infty}: \mathbf{E}^{\infty}} .
$$

This expression is general, but since we have restricted ourselves to the study of dilute suspensions $(\varphi \ll 1), \mathbf{M}_{E S}$ corresponds only to the "self" parts of the grand mobility tensor. In the limit that the gap between the walls becomes infinite in extent, the inverse mobility coupling between rate of strain and stresslet becomes an isotropic tensor proportional to $\frac{20}{3} \pi \eta a^{3}$ and we recover the Einstein viscosity, $\eta^{*} / \eta=1$ $+\frac{5}{2} \varphi$. Thus, we write the viscosity as

$$
\frac{\eta^{*}}{\eta}=1+\frac{5}{2} \varphi\left[1+Z\left(\frac{H}{a}\right)\right],
$$

where

$$
Z\left(\frac{H}{a}\right)=\frac{\mathbf{E}^{\infty}:\left\langle\mathbf{M}_{E S}^{-1}\right\rangle: \mathbf{E}^{\infty}}{\frac{20}{3} \pi \eta a^{3} \mathbf{E}^{\infty}: \mathbf{E}^{\infty}}-1 .
$$

Shortly after Einstein made his calculation of the viscosity of a dilute suspension, Guth and Simha ${ }^{31}$ attempted to include the effects of channel walls on the suspension viscosity. In their approach, the effects of each wall were superimposed $a$ la Oseen, ${ }^{13}$ and the resulting particle stresslet was calculated while assuming the particles were evenly distributed in the channel. With this approximation, they found that

$$
Z\left(\frac{H}{a}\right)=\frac{5}{16}\left(\frac{H}{a}\right)\left[\left(\frac{H}{a}\right)^{2}-1\right]^{-1} .
$$

Like Guth and Simha, we assume that the dilute suspension is uniformly distributed between the walls and calculate the average of $\mathbf{M}_{E S}^{-1}$ as

$$
\left\langle\mathbf{M}_{E S}^{-1}\right\rangle=\frac{1}{1-2(a / H)} \int_{a / H}^{1-a / H} \mathbf{M}_{E S}^{-1}\left(\Xi ; \frac{H}{a}\right) d \Xi .
$$

To a first approximation for widely separated channel walls, and parallel plate rheometry (i.e., $\mathbf{E}_{i j}^{\infty}=\delta_{i 1} \delta_{j 2}+\delta_{i 2} \delta_{j 1}$ ), the contribution to the viscosity can be written quite simply, viz.,

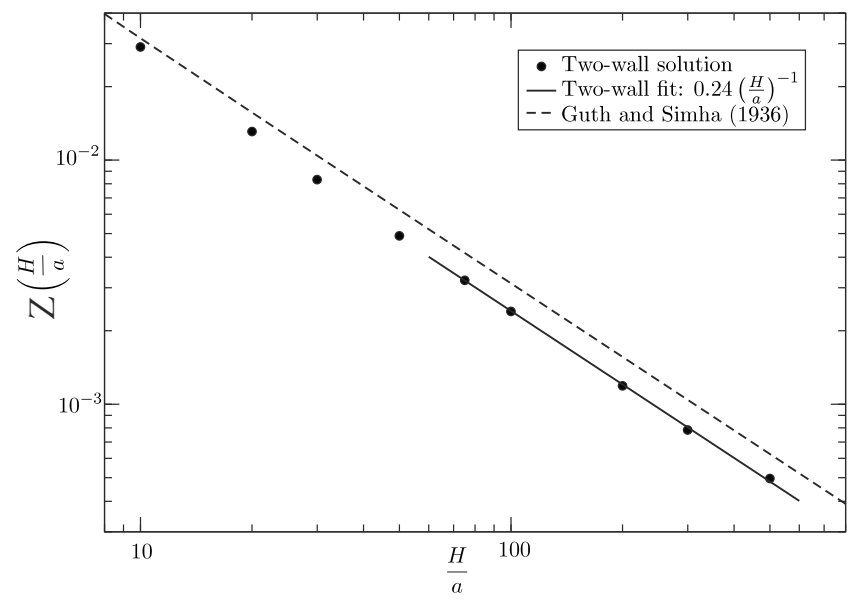

FIG. 13. The additional contribution to the viscosity of a dilute suspension, $\eta^{*} / \eta=1+\frac{5}{2} \varphi[1+Z(H / a)]$, is plotted against the separation between the channel walls. The superposition approximation due to Guth and Simha is also plotted.

$$
Z\left(\frac{H}{a}\right)=\frac{20}{9}\left(\frac{a}{H}\right)^{3} \frac{1}{1-2(a / H)} \int_{a / H}^{1-a / H} g_{3}(\Xi) d \Xi .
$$

For wide channels $(H / a \gg 1)$, the dominant contributions to the above integral are near $\Xi=0$ and $\Xi=1$, where $g_{3}(\Xi)$ scales like the single wall values, $\Xi^{-3}$ and $(1-\Xi)^{-3}$, respectively. Therefore, in this regime we predict that $Z(H / a)$ scales like $a / H$, which is the same scaling predicted by Guth and Simha. In Fig. 13 we plot the additional contribution to the viscosity of a dilute suspension as well as the result due to Guth and Simha. We find that this additional contribution is always smaller than that predicted by superposition. Note that another measure of this same quantity was made recently by Zurita-Gotor et $a .^{32}$ They compute the viscosity over a narrower range of channel widths which do not overlap with those presented here. Similarly, our analysis is not applicable to the narrow channels they considered. Comparison of the two results is therefore difficult. Our aim in this section was to compare superposition with the Fourier transform solution in a regime where the particles spend little time in the lubrication zone near the wall. Our result is quite different from this other calculation.

\section{EXTENSIONS AND CONCLUSIONS}

In this article we computed the exact reflection of various multipolar velocity fields off of the parallel walls of a channel. These were combined with different Faxén formulas to generate the far-field mobility tensor for a single spherical particle in a channel. We found that the mobility could be written in terms of inverse powers of the channel width multiplied by functions of the fractional distance of the particle across the channel. These functions matched predictions for a particle in the middle of the channel and a quarter of the way across the channel made by Faxén in his dissertation nearly a century ago. ${ }^{11}$ The form we have used to represent these mobility functions is especially useful for computational studies since the functions of the fractional distance 
across the channel can be tabulated and used for channels of arbitrary width. The tables for the six mobility tensors discussed within are available from the editorial office. Using these functions we made additional predictions of the single particle fall speed both along and perpendicular to the channel walls. We also calculated the rotation rate of a particle sedimenting along the channel and found that the direction of rotation reverses near the midline of sufficiently wide channels. We calculated the Brownian drift velocity for a single particle and the correction to the Einstein viscosity for a dilute suspension in a wide channel.

While the method described deals only with computations for single particles, the same approach may be extended to the study of suspensions with interparticle hydrodynamic interactions. This could take the form of either a traditional Stokesian dynamics simulation or an accelerated Stokesian dynamics simulation. The key point is that the reciprocal space representation of the hydrodynamic interactions is the most natural way to represent computationally the far-field many-bodied interactions amidst a suspension. The inverse transformation may not even be necessary if a simulation is properly constructed to represent the suspension in a set of periodic cells. Using the Poisson summation formula, one can show that adding all the interactions to a single particle and its periodic images is the same as summing over the reciprocal space representation of the interactions with a Fourier exponential weighting. ${ }^{33}$ One important piece to consider here, however, is that this summation is slowly converging in general. To make things computationally efficient, the domain of interactions must be split into two regions: one over which short-range, real space interactions dominate and another over which long-range, reciprocal space interactions are most important. This is precisely the Ewald summation technique that turns one slowly converging summation into two rapidly converging ones. In the case of the short-range interactions, in all likelihood it will be sufficient to represent the channel walls as a superposition of two single walls only, which are already available. ${ }^{4}$ While for the long-range interactions, the full reciprocal space two wall solution will be needed. This approach will avoid the costly inversion of the solutions to the Stokes flow equations from the reciprocal space back to the real space while still accurately reflecting the condition of no-slip on each of the walls. An approach like this one could overcome some of the difficulties that make simulations of particles in a channel challenging.

It is also possible to extend the techniques described here to the study of other particle and boundary geometries. In our approach, we only worked out the reflection of the Stokeslet off two walls explicitly. All the higher order reflections came from a multipole expansion of the boundary integral solution to the Stokes equations. A similar expansion may be performed for particles of any geometry; though, it will not truncate as succinctly as when the force density is expanded about a spherical surface. Nevertheless, this result combined with an appropriate Faxén type formula for that particular particle geometry will generate the grand mobility tensor. Similarly, if the reflection of the Stokeslet is found or is known in some other boundary geometry (e.g., a cylindrical channel ${ }^{34}$ or a spherical container ${ }^{35}$ ), then calculation of the grand mobility tensor is as simple as choosing the proper multipole expansion and applying the Faxén formula. No doubt the results will be similar for particles that are roughly spherical in shape as Stokes flow is rather insensitive to geometric details. However, for extreme shapes like slender bodies, this approach offers an interesting possibility for studying their dynamics under confinement.

In closing, we want to emphasize the simplicity of this approach for treating the hydrodynamics of a particle in a confining geometry. The higher level perspective brought by the grand mobility tensor takes the complicated problem of determining the resistance of a particle and turns it into a hierarchical method of reflections type procedure, which has a direct physical connection to the different force moments on the particle. In this article, we have computed once and for all the first nine components of the grand mobility tensor for a spherical particle in a parallel wall channel. While there are infinitely many higher order contributions to that tensor, those first nine have proven quite effective in other situations for characterizing the dynamics of particles in suspensions. Similarly, these tensors provide a simple and therefore rather useful way to estimate the motion of a small particle in a channel. By tabulating these functions and separating out the length scale set by the channel width, we have enabled the rapid computation of single particle and dilute suspension dynamics in a channel. ${ }^{36}$

\section{APPENDIX: SOLUTION TO THE STOKES EQUATIONS FOR ARBITRARY BOUNDARY CONDITIONS ON THE CHANNEL WALLS}

In this appendix we conclude the derivation of the solution to the Stokes flow equations with arbitrary boundary conditions on a set of parallel walls. We designate the lower wall as $r_{3}=r_{l}$ and the upper wall as $r_{3}=H+r_{l}$ and write the boundary conditions on the flow as

$$
\begin{aligned}
\hat{\mathbf{v}}^{L}= & \mathbf{A} e^{-r_{l} k}+\mathbf{B} e^{r_{l} k}+\frac{1}{4 \eta k^{2}}\left[A \mathbf{d}\left(2 r_{l} k+1\right) e^{-r_{l} k}\right. \\
& \left.+B \overline{\mathbf{d}}\left(2 r_{l} k-1\right) e^{r_{l} k}\right], \\
\hat{\mathbf{v}}^{U}= & \mathbf{A} e^{-\left(H+r_{l}\right) k}+\mathbf{B} e^{\left(H+r_{l}\right) k}+\frac{1}{4 \eta k^{2}} \\
& \times\left\{A \mathbf{d}\left[2\left(H+r_{l}\right) k+1\right] e^{-\left(H+r_{l}\right) k}+B \overline{\mathbf{d}}\right. \\
& \left.\times\left[2\left(H+r_{l}\right) k-1\right] e^{\left(H+r_{l}\right) k}\right\} .
\end{aligned}
$$

The solution to these equations is more conveniently written in the typical matrix-vector form, 


$$
\left(\begin{array}{l}
\mathbf{A} \\
\mathbf{B}
\end{array}\right)=\frac{1}{2}[\operatorname{coth}(H k)-1] e^{H k} \times\left[\begin{array}{cc}
e^{\left(H+r_{l}\right) k} & -e^{r_{l} k} \\
-e^{-\left(H+r_{l}\right) k} & e^{-r_{l} k}
\end{array}\right]\left(\begin{array}{c}
\hat{\mathbf{v}}^{L}-\frac{1}{4 \eta k^{2}}\left[A \mathbf{d}\left(2 r_{l} k+1\right) e^{-r_{l} k}+B \overline{\mathbf{d}}\left(2 r_{l} k-1\right) e^{r_{l} k}\right] \\
\hat{\mathbf{v}}^{U}-\frac{1}{4 \eta k^{2}}\left\{A \mathbf{d}\left[2\left(H+r_{l}\right) k+1\right] e^{-\left(H+r_{l}\right) k}+B \overline{\mathbf{d}}\left[2\left(H+r_{l}\right) k-1\right] e^{\left(H+r_{l}\right) k}\right\}
\end{array}\right) .
$$

We still need to determine the coefficients $A$ and $B$, but since the velocity field is divergence free, Eq. (25) sets the relationship between these coefficients and the vectors $\mathbf{A}$ and $\mathbf{B}$. Applying this relationship and solving for the unknown coefficients yields the following:

$$
\begin{aligned}
\left(\begin{array}{l}
A \\
B
\end{array}\right)= & \left(\frac{2 \eta}{1+2(H k)^{2}-\cosh (2 H k)}\right) \\
& \times\left(\begin{array}{cc}
-\sinh (H k) & H k e^{k\left(H+2 r_{l}\right)} \\
H k e^{-k\left(H+2 r_{l}\right)} & -\sinh (H k)
\end{array}\right) \\
& \times\left(\begin{array}{c}
\mathbf{d} \cdot\left(\hat{\mathbf{v}}^{L} e^{\left(H+r_{l}\right) k}-\hat{\mathbf{v}}^{U} e^{r_{l} k}\right) \\
\overline{\mathbf{d}} \cdot\left(\hat{\mathbf{v}}^{L} e^{-\left(H+r_{l}\right) k}-\hat{\mathbf{v}}^{U} e^{-r_{l} l^{k}}\right)
\end{array}\right) .
\end{aligned}
$$

This completes the derivation of the solution of the Stokes equations in the space bounded by parallel walls with arbitrary boundary conditions. Of course, this is only the wave space solution to the problem. The inversion of these results to find the real space solution will depend on the details of the vectors $\hat{\mathbf{v}}^{L}$ and $\hat{\mathbf{v}}^{U}$. For even simple boundary conditions, this process can be quite difficult, and it is necessary to combine a clear physical picture of the problem at hand with a detailed knowledge of integral transform techniques. In the above article, we illustrate the inversion of the reflection of a Stokeslet, but only at the place where the reflected flow field corresponds to the location of the point force. The techniques employed are useful for calculating hydrodynamic interactions among particles although perhaps less useful for imaging the flow field or making more general calculations.

${ }^{1}$ D. Leighton and A. Acrivos, "Measurement of shear-induced selfdiffusion in concentrated suspensions of spheres," J. Fluid Mech. 177, 109 (1987).

${ }^{2}$ T. Squires and S. Quake, "Microfluidics: Fluid physics at the nanoliter scale," Rev. Mod. Phys. 77, 977 (2005).

3 J. C. Crocker and D. G. Grier, "When like charges attract: The effects of geometrical confinement of long-range colloidal interactions," Phys. Rev. Lett. 77, 1897 (1996).

${ }^{4}$ J. W. Swan and J. F. Brady, "Simulation of hydrodynamically interacting particles near a no-slip boundary," Phys. Fluids 19, 113306 (2007).

${ }^{5} \mathrm{O}$. D. Velev and K. H. Bhatt, "On-chip micromanipulation and assembly of colloidal particles by electric fields," Soft Matter 2, 738 (2006).

${ }^{6} \mathrm{E}$. Verpoorte, "Microfluidic chips for clinical and forensic analysis," Electrophoresis 23, 677 (2002).

${ }^{7}$ S. K. Sia and G. M. Whitesides, "Microfluidic devices fabricated in poly(dimethylsiloxane) for biological studies," Electrophoresis 24, 3563 (2003).

${ }^{8}$ R. D. Astumian and P. Hänggi, "Brownian motors," Phys. Today 55(11), 33 (2002).

${ }^{9}$ A. Gopinathan and D. G. Grier, "Statistically locked-in transport through periodic potential landscapes," Phys. Rev. Lett. 92, 130602 (2004).

${ }^{10}$ M. P. MacDonald, G. C. Spalding, and K. Dholakia, "Microfluidic sorting in an optical lattice," Nature (London) 426, 421 (2003).

${ }^{11}$ H. Faxen, Ark. Mat., Astron. Fys. 17, 1 (1923); Ph.D. dissertation, Upp- sala University, 1921.

${ }^{12}$ J. Happel and H. Brenner, Low Reynolds Number Hydrodynamics, 2nd ed. (Prentice-Hall, Englewood Cliffs, NJ, 1986).

${ }^{13}$ C. W. Oseen, "Neuere Methoden und Ergebnisse in der Hydrodynamik," Ph.D. dissertation, Akademische Verlagsgesellschaft, 1928.

${ }^{14}$ J. R. Blake and A. T. Chwang, "Fundamental singularities of viscous flow," J. Eng. Math. 8, 23 (1974).

${ }^{15}$ N. Liron and S. Mochon, "Stokes flow for a Stokeslet between two parallel flat plates,” J. Eng. Math. 10, 287 (1976).

${ }^{16}$ L. J. Durlofsky and J. F. Brady, "Dynamic simulation of bounded suspensions of hydrodynamically interaction particles," J. Fluid Mech. 200, 39 (1989).

${ }^{17}$ P. R. Nott and J. F. Brady, "Pressure-driven flow of suspensions: Simulation and theory," J. Fluid Mech. 275, 157 (1994).

${ }^{18}$ A. Singh and P. R. Nott, "Normal stresses and microstructure in bounded sheared suspensions via Stokesian dynamics simulations," J. Fluid Mech. 412, 279 (2000).

${ }^{19}$ B. Cichocki, R. B. Jones, R. Kutteh, and E. Wajnryb, "Friction and mobility for colloidal spheres in Stokes flow near a boundary: The multipole method and applications," J. Chem. Phys. 112, 2548 (2000).

${ }^{20} \mathrm{~S}$. Bhattacharya and J. Blawzdziewicz, "Image system for Stokes-flow singularity between two parallel planar walls," J. Math. Phys. 43, 5720 (2002).

${ }^{21}$ R. B. Jones, "Spherical particle in Poiseuille flow between planar walls," J. Chem. Phys. 121, 483 (2004).

${ }^{22}$ P. Ganatos, S. Weinbaum, and R. Pfeffer, "A strong interaction theory for the creeping motion of a sphere between plane parallel boundaries. Part 1. Perpendicular motion,” J. Fluid Mech. 99, 739 (1980).

${ }^{23}$ M. E. Staben, A. Z. Zinchenko, and R. H. Davis, "Motion of a particle between two parallel plane walls in low-Reynolds-number Poiseuille flow," Phys. Fluids 15, 1711 (2003).

${ }^{24}$ E. R. Dufresne, D. Altman, and D. G. Grier, "Brownian dynamics of a sphere between parallel walls," Europhys. Lett. 53, 264 (2001).

${ }^{25}$ S. Kim and S. J. Karrila, Microhydrodynamics, 2nd ed. (Dover, Mineola, NY, 1991).

${ }^{26}$ L. J. Durlofsky, J. F. Brady, and G. Bossis, "Dynamic simulation of hydrodynamically interacting particles," J. Fluid Mech. 180, 21 (1987).

${ }^{27}$ G. Bossis, A. Meunier, and J. D. Sherwood, "Stokesian dynamics simulations of particle trajectories near a plane," Phys. Fluids A 3, 1853 (1991).

${ }^{28}$ T. N. Phung, J. F. Brady, and G. Bossis, "Stokesian dynamics simulations of Brownian suspensions," J. Fluid Mech. 313, 181 (1996).

${ }^{29} \mathrm{G}$. K. Batchelor, "Brownian diffusion of particles with hydrodynamic interaction," J. Fluid Mech. 74, 1 (1976).

${ }^{30}$ D. L. Ermak and J. D. McCammon, "Brownian dynamics with hydrodynamic interactions," J. Chem. Phys. 69, 1352 (1978).

${ }^{31}$ E. Guth and R. Simha, "Untersuchungen über die Viskosität von Suspensionen und Lösungen. 3. Über die Viskosität von Kugelsuspensionen," Colloid Polym. Sci. 74, 266 (1936).

${ }^{32}$ M. Zurita-Gotor, J. Blawzdziewicz, and E. Wajnryb, "Motion of a rod-like particle between parallel walls with application to suspension rheology," J. Rheol. 51, 71 (2007).

${ }^{33}$ C. W. J. Beenakker, "Ewald sum of the Rotne-Prager tensor," J. Chem. Phys. 85, 1581 (1986).

${ }^{34}$ N. Liron and R. Shahr, "Stokes flow due to a Stokeslet in a pipe," J. Fluid Mech. 86, 727 (1978).

${ }^{35} \mathrm{C}$. Maul and S. Kim, "Image of a point force in a spherical container and its connection to the Lorentz reflection formula," J. Eng. Math. 30, 119 (1996).

${ }^{36}$ See supplementary material at http://dx.doi.org/10.1063/1.3487748 for tables of all mobility functions described in this article for values of the fractional distance across the channel spaced $10^{-3}$ apart. 